\title{
Information Content of Very High Resolution SAR Images: Study of Feature Extraction and Imaging Parameters
}

\author{
Corneliu Octavian Dumitru and Mihai Datcu, Fellow, IEEE
}

\begin{abstract}
In this paper, we propose to study the dependence of information extraction technique performance on synthetic aperture radar (SAR) imaging parameters and the selected primitive features (PFs). The evaluation is done on TerraSAR-X data, and the interpretation is realized automatically. In the first part of this paper (use case I), the following issues are analyzed: 1) finding the optimal TerraSAR-X products and their limits of variability and 2) retrieving the number of categories/classes that can be extracted from the TerraSAR-X images using the PFs (gray-level co-occurrence matrix, Gabor filters, quadrature mirror filters, and nonlinear short-time Fourier transform). In the second part of this paper (use case II), we investigate the invariance of the products with the orbit direction and incidence angle. On the one hand, the results show that using ascending looking is better than using descending looking with an average accuracy increase of $7 \%-8 \%$, approximately. On the other hand, the classification accuracy for the incidence angle varies from a lower value of the incidence to an upper value of the incidence angle (depending on the sensor range) with $4 \%-5 \%$. The test sites are Venice (Italy), Toulouse (France), Berlin (Germany), and Ottawa (Canada) and are covering as much as possible the huge diversity of modes, types, and geometric resolution configuration of the TerraSAR-X. For the evaluation of all these parameters (resolution, features, orbit looking, and incidence angle), the support-vector-machine classifier is considered. To evaluate the accuracy of the classification, the precision/recall metric is calculated. The first contribution of this paper is the evaluation of different PFs (proposed in the literature for different types of images) and adaptation of these for SAR images. These features are compared (based on the accuracy of the classification) for the first time for a multiresolution pyramid specially built for this purpose. During the evaluation, all the classes were annotated, and a semantic meaning was defined for each class. The second main contribution of this paper is the evaluation of the dependence on the patch size, orbit direction, and incidence angle of the TerraSAR-X. This type of evaluation has not been systematically investigated so far. For the evaluation of the optimal patch, two different patch sizes were defined, with the constrained that the size on ground needs to cover a minimum of one object (e.g., $200 \times 200 \mathrm{~m}$ on ground). This patch size depends also on the parameters of the data such as resolution and pixel spacing. The investigation of orbit looking and incidence angle is very important for indexing large data sets that has a higher
\end{abstract}

Manuscript received July 11, 2012; revised December 10, 2012 and April 19, 2013; accepted May 7, 2013. Date of publication July 5, 2013; date of current version July 22,2013

The authors are with the Remote Sensing Technology Institute (IMF), Earth Observation Center, German Aerospace Center (DLR), 82234 Wessling, Germany (e-mail: corneliu.dumitru@dlr.de; mihai.datcu@dlr.de).

Color versions of one or more of the figures in this paper are available online at http://ieeexplore.ieee.org.

Digital Object Identifier 10.1109/TGRS.2013.2265413 variability of these two parameters. These parameters influence the accuracy of the classification (e.g., if the incidence angle is closer to the lower bounds or closer to the upper bound of the satellite sensor range).

Index Terms-Annotation, Fourier, Gabor, gray-level cooccurrence matrix (GLCM), incidence angle, orbit direction, patch, primitive features (PFs), quadrature mirror filter (QMF), semantic, support vector machine (SVM), TerraSAR-X.

\section{INTRODUCTION}

$\mathbf{P}$ RESENTLY, Earth observation (EO) satellites acquire huge volumes of high-resolution images, very much overpassing the capacity of the users to access the information content of the acquired data. In addition to the existing methods for EO, new methods and tools are needed in order to extract the information and to help to discover the information hidden in large EO image repositories.

The first major consideration in the development of the system is the determination of relevant content that will be used when the images are classified.

For synthetic aperture radar (SAR) images, mainly for the sensors with resolution between 1 and $10 \mathrm{~m}$ such as TerraSAR-X, TanDEM-X, and Sentinel 1, there is a stringent need to design algorithms for primitive feature $(\mathrm{PF})$ in order to estimate and to characterize these images.

In the area of image analysis, the quality of the estimated PFs is of crucial importance for any further automated or semiautomated interpretation in the case of query engines and image information mining (IIM) impacting the overall performance.

For high-resolution satellite images, we proposed to use a method that is based on patches [9] (which are small images of about $200 \times 200$ pixels tiled from the original image) in order to extract the relevant information. The methods proposed until now in the literature are pixel-based methods; these methods do not capture the contextual information, and the global features describing the overall properties of images are not accurate enough.

Among the typical PFs, different quality metrics can be enumerated: the variance of the estimated parameters and its dependence on $S / N$ [signal-to-noise ratio (SNR)] and analyzing window, the influence of the assumptions of stationarity, the radiometric variability, the invariance to rotation or/and scaling transforms, the influence of occlusions or shadows, etc.

TerraSAR-X has a huge diversity of modes (StripMap (SM), SpotLight (SL), and ScanSAR), types (complex, detected, and 


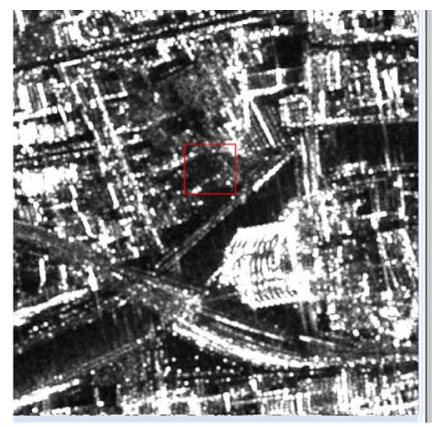

High resolution Spotlight mode (HS)

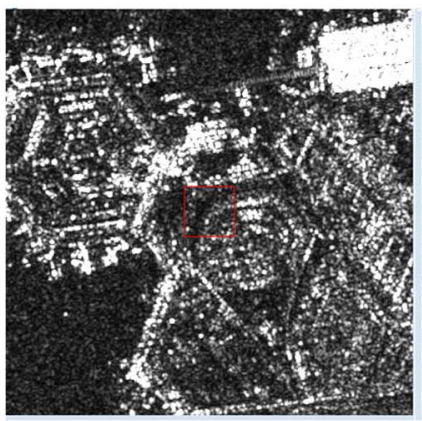

Spatially Enhanced (SE)

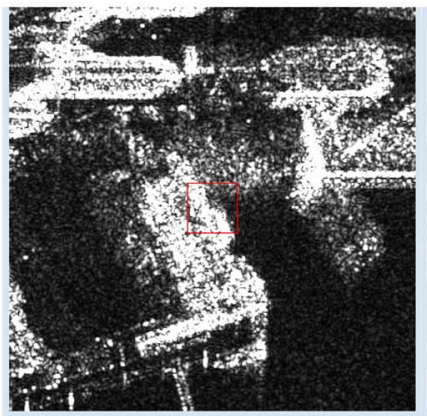

Multi-look Ground Detected (MGD)

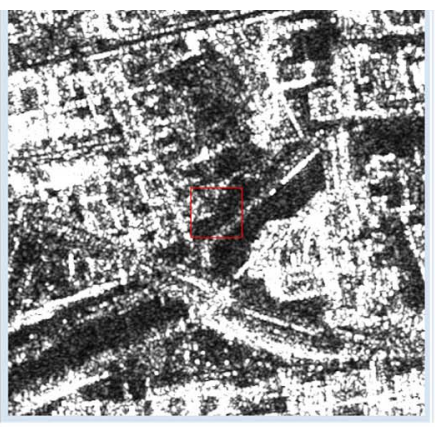

StripMap mode (SM)

(a)
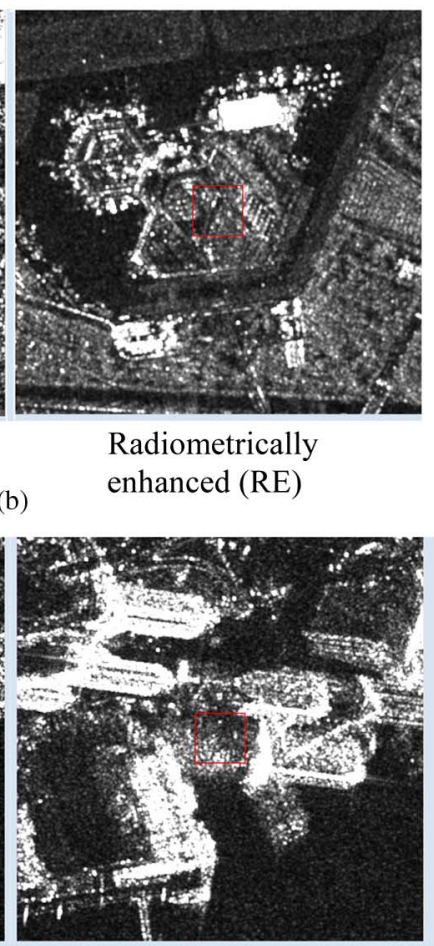

Geo-coded Ellipsoid range Corrected (GEC)

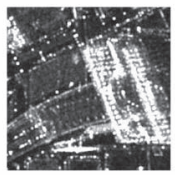

Resolution $6.5 \mathrm{~m}$ Pixel spacing $2.75 \mathrm{~m}$

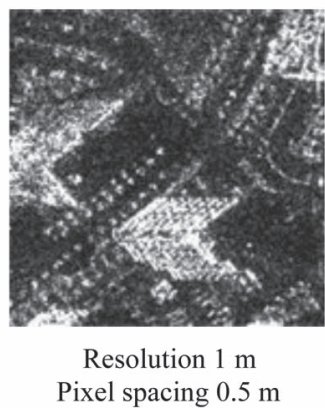

(a)

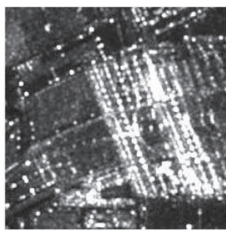

Resolution $2.9 \mathrm{~m}$

Pixel spacing $1.25 \mathrm{~m}$

(b)

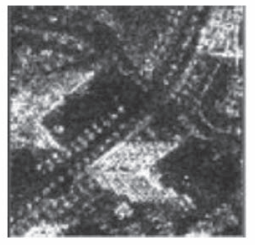

Resolution $2.9 \mathrm{~m}$

Pixel spacing $1.25 \mathrm{~m}$
Fig. 2. Examples showing (a-top of the figure) the difference of patch size for two products (GEC-RE with SM mode and HS mode) having different resolutions and pixel spacings and (b-bottom of the figure) the influence of resolution and pixel spacing on the size of a patch for two TerraSAR-X products (GEC-SE and GEC-RE)

During the evaluation, the TerraSAR-X products that exist in our data set are tiled in patches at different sizes (number of pixels), depending on the characteristics of the TerraSAR-X product, in order to have the same area of $200 \times 200 \mathrm{~m}$ covered on ground. The scale of buildings is about $200 \times 200 \mathrm{~m}$, and the size is chosen in order to have about one object inside of each patch. The parameters that influence the size of the patch are the resolution and the pixel spacing, and based on this, the tiled patches are having different sizes. In the next figure [see Fig. 2(a)], we considered two patches tiled from GEC products, RE with an SM mode and an HS mode. The sizes of these two patches are different because the parameters of these two tiled products are as follows:

1) resolution-6.5 $\mathrm{m}$ for $\mathrm{SM}$ and $2.9 \mathrm{~m}$ for $\mathrm{HS}$;

2) pixel spacing-2.75 $\mathrm{m}$ for $\mathrm{SM}$ and $1.25 \mathrm{~m}$ for $\mathrm{HS}$.

In this paper, we study the sensitivity of the information extraction process with respect to different TerraSAR-X data parameters.

1) First, the invariance of the data with the resolution and pixel spacing of TerraSAR-X images. An example is presented in Fig. 2 (top part of the figure), and the second one is presented in Fig. 2 (bottom of the figure), where two products having different geographical configurations are compared based on the influence of the resolution and pixel spacing on the patch size that covers the same area on ground. $\rightarrow$ sensitivity with respect to the resolution and pixel spacing.

2) Second, the sensibility of TerraSAR-X data with the orbit direction. In Fig. 3, such an example is presented in order to understand the difference between the data acquired with an ascending looking and data acquired with a descending looking. $\rightarrow$ sensitivity with respect to the orbit direction. corrected (GEC). 


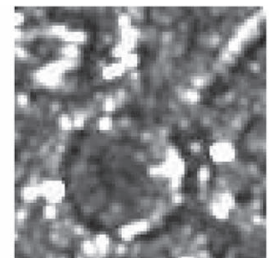

Orbit with ascending looking

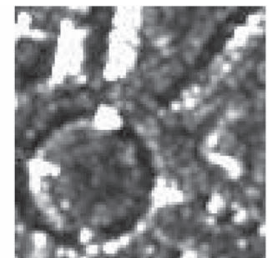

Orbit with descending looking

Fig. 3. Example presenting the difference between the ascending and the descending orbit looking for two TerraSAR-X products (GEC-RE with SM)

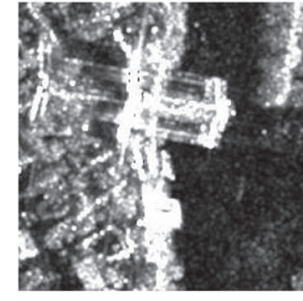

Incidence angle $27^{\circ}$

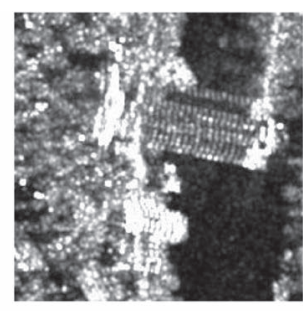

Incidence angle $41^{\circ}$

Fig. 4. Example of two patches tiled from TerraSAR-X products (GEC-R with HS) that were acquired with different values of the incidence angle: $27^{\circ}$ (close to the lower bound of the sensor range) and $41^{\circ}$ (close to the upper bound of the sensor range).
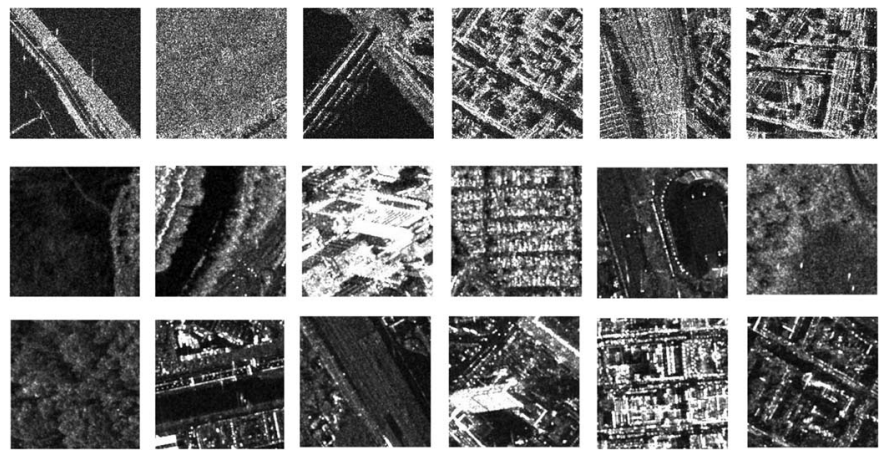

Fig. 5. Typical patches/classes that can be identified in a high-resolution TerraSAR-X data set.

3) Finally, the sensibility of TerraSAR-X data with the incidence angle. In the next figure (see Fig. 4), two examples are presented where the data were acquired with the same orbit looking (e.g., descending) and with two different values of the incidence angle (e.g., $27^{\circ}$ and $41^{\circ}$ ) covering the min-max range of the sensor. $\rightarrow$ sensitivity with respect to the incidence angle.

For high-resolution SAR images, the diversity of the classes [2] that can be retrieved from the product image is higher than the case of lower or medium resolution. This is demonstrated in Fig. 5 with some examples, where the diversity of the content that exists in the patches selected from our data set is shown.

Two data sets are specially created in order to answer the questions about the sensitivity of the SAR images related to the parameters of the data (e.g., resolution, pixel spacing, orbit direction, and incidence angle).

The flowchart of the system used to investigate the dependence of different parameters of the product and features extracted from the data is presented in Fig. 6. We begin by tiling the product image into patches whose size depends on the parameters of the data (e.g., resolution and pixel spacing). For each patch, a set of features is extracted [e.g., gray-level cooccurrence matrix (GLCM), Gabor filters (GAFs), quadrature mirror filters (QMFs), and nonlinear short-time Fourier transform (STFT) (NSFT)], and they are grouped in classes using a classifier. For each class, a semantic meaning [3] is chosen based on the dominant content of the patch and using as visual support the ground truth of Google Earth. For each evaluation, we calculate the precision/recall, and after that, we select the optimal parameters.

Based on previous examples shown in Figs. 1-5 for each application, an optimum set of parameters (products, orbit direction, incidence angle, and features) needs to be selected from the huge existing variety.

The knowledge to be gained and the results that we will obtain during this evaluation can be used for specific applications that include the following: urbanism, automatic target recognition and man-made structure recognition, cartography, rapid mapping, and payload ground segment (PGS) [4] archive catalog design.

The organization of this paper is as follows: Section II presents the basic TerraSAR-X products, and based on their characteristics, we proposed a configuration of the TerraSAR-X product to be used for evaluation. Using this configuration, two test data sets are built and described separately in the next sections for each use case. Section III explains in detail the following: 1) the preprocessing of the data before being used for evaluation; 2) the feature extraction methods to be applied such as statistical methods (GLCM) and spectral methods (e.g., GAFs, QMFs, and NSFT); and, finally, 3) the methodology to be used for the evaluation of two use cases. The first use case (see Section IV) investigates the dependence of the resolution, pixel spacing, and PF (for all four methods) for a multiresolution pyramid and proposes a configuration of the data that can be used further for other evaluation. This configuration is referring to the type, mode, and geometric resolution configuration of the product and features to be used in order to describe the data. The second use case (see Section V) gives the optimal patch size, orbit direction, and incidence angle for a higher accuracy of the classification. Using the knowledge from the first use case (e.g., standard product, RE, and features GAFs or QMFs), we built a new data set in order to find the following: 1) the optimal patch size for products having SM mode or HS mode; 2) the orbit direction considering the same product (e.g., SM mode), the same area, and the optimal size of the patch previously defined; and 3) the incidence angle having the same orbit direction (e.g., ascending/descending), mode (e.g., HS mode), and optimal patch size. In the end of this section, we will have an idea about what configuration of product to use and what parameters (orbit direction and incidence angle) to be selected. This paper ends with conclusions and future work.

\section{EO TERRASAR-X PRODUCTS}

In this section, we discuss the EO data represented by the TerraSAR-X products that are intend to be used in our evaluation. TerraSAR-X is a German radar satellite, and it operates in the X-band and is a side-looking SAR based on active 


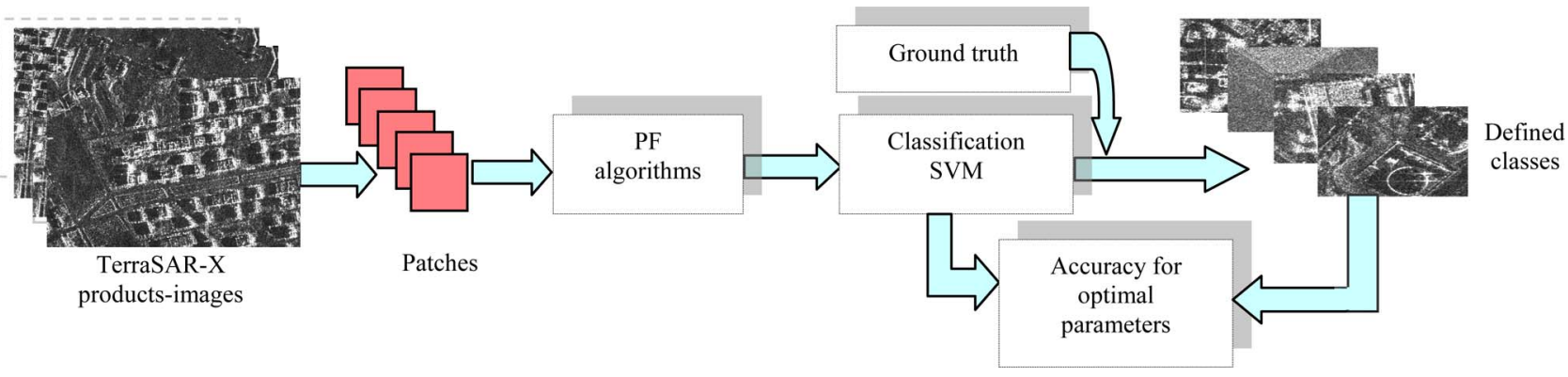

Fig. 6. Proposed methodology: Input SAR images are tiled into patches, and the PFs are computed for each patch, classified, and grouped in classes using for visual support the ground truth of Google Earth. After the classes are defined, the procedure is repeated in order to identify the best PF and the optimal parameters (resolution, pixel spacing, patch size, and some other parameters available in the metadata of TerraSAR-X product such as orbit direction and incidence angle).

phased array antenna technology [1]. The TerraSAR-X product contains two components: the image (EO TerraSAR-X image) and the descriptors of the acquisition system (EO TerraSAR-X XML).

The individual polarization layers of the image data of projected products are given as separated files in the GeoTIFF file format [6] in unsigned $16 \mathrm{~b}$ (for detected data)/32 $\mathrm{b}$ (for complex data) representation and a subset of commonly used tags [1].

The TerraSAR-X annotation file contains a complete description of the TerraSAR-X product components, and it is considered as metadata source [1]. Data types, valid entries, and allowed attributes are defined in detail for each element in this file.

In Section II-A, we described the different types of TerraSAR-X products/images, while in Section II-B, we presented the selected products for our investigation.

\section{A. TerraSAR-X L1b Products}

The TerraSAR-X products are "Level 1b products" (L1b) [1] which are the operational products offered to scientific customers. The products are generated by the TerraSAR-X multimode SAR processor (TMSP) available at the German Aerospace Center (DLR).

The basic products are in a huge diversity of modes (SM, SL, and ScanSAR), types (complex, detected, and geocoded), and geometric resolution configurations (SE or RE products) requiring product annotation in an extensible and dynamic format. The XML format has been selected for this purpose [1].

The TerraSAR-X instrument acquisition operates in three modes: SM, SL, and ScanSAR.

The SM mode is the basic-standard SAR imaging mode as known (e.g., ERS-1). The ground range is $30 \mathrm{~km}$ for a single polarization, and the nominal $\mathrm{L} 1 \mathrm{~b}$ product length is about $50 \mathrm{~km}$. The azimuth resolution of the product is $3.3 \mathrm{~m}$, and the full range of the incidence angle is between $20^{\circ}$ and $45^{\circ}$. For a ground range resolution of $1.7 \mathrm{~m}$, the incidence angle is about $45^{\circ}$, and for $3.49 \mathrm{~m}$, the incidence angle is $20^{\circ}$.

The SL mode uses phased array beam steering in the azimuth direction to increase the illumination time, i.e., the size of the synthetic aperture. Two variants of the SL mode are designed with different values for the azimuth resolution and scene size. For both variants, the ground range resolution is between $1.48 \mathrm{~m}$ for the incidence angle equal to $55^{\circ}$ and $3.49 \mathrm{~m}$ for the incidence angel equal to $20^{\circ}$.
The HS mode is designed for an azimuth resolution of $1.1 \mathrm{~m}$, resulting in an azimuth scene size of $5 \mathrm{~km}$ by $10 \mathrm{~km}$ in ground range.

For the second variant, the SL mode, the beam steering velocity is lower than that of the HS mode resulting in reduced azimuth resolution $(1.7 \mathrm{~m})$ and increased azimuth scene extension (10 km in azimuth by $10 \mathrm{~km}$ in ground range).

For the ScanSAR mode, the electronic antenna elevation steering is used to switch after bursts of pulse between swathers with different incidence angles. This mode is designed for large areas of $100 \mathrm{~km}$ where four SM beams are combined. The azimuth resolution of the product is $18.5 \mathrm{~m}$, and the ground range is between $1.70 \mathrm{~m}$ (for an incidence angle of $45^{\circ}$ ) and $3.49 \mathrm{~m}$ (for an incidence angle of $20^{\circ}$ ).

Note that the ground range resolution depends on the incidence angle: For a high value of the incidence angle (flat view), you will have a good ground range resolution, and for a smaller value of the incidence angle (steep or vertical view), you will have a bad ground range resolution.

The SAR data (which come directly from the satellite) are processed to basic products by TerraSAR-X TMSP, which generates a set of images depending on the geometric and radiometric resolution as well as on the geometric projection and data representation. Fig. 7 summarizes the TerraSAR-X basic products [8].

The SAR images can have the range and azimuth resolution different depending on the image mode and incidence angle. In order to obtain equal resolution in the range and azimuth, a multilooking is applied to equalize these two resolutions in the case of SE.

The RE product is optimized with respect to radiometry [1]. The range and azimuth resolution are decreased significantly in order to reduce the speckle by averaging six looks in order to obtain a radiometric resolution of $1.5 \mathrm{~dB}$. The SNR decrease with larger incidence angles is also considered assuming backscatters of $-6 \mathrm{~dB}$ at $20^{\circ}$ and $-12 \mathrm{~dB}$ at $50^{\circ}$. For a lower resolution, the required pixel spacing can be reduced with the impact of decreasing the product data size.

The TerraSAR-X allows a selection among different geometrical projections and data representations. The single look slant range (SSC) product is the single look complex product of the focused radar signal. The data have complex numbers, and the pixels are spaced equidistant in azimuth and in slant range.

The MGD product is a multilook detected product with reduced speckle and equal resolution on ground. The MGD preserves the geometry of the observed objects without geographical orientation. 


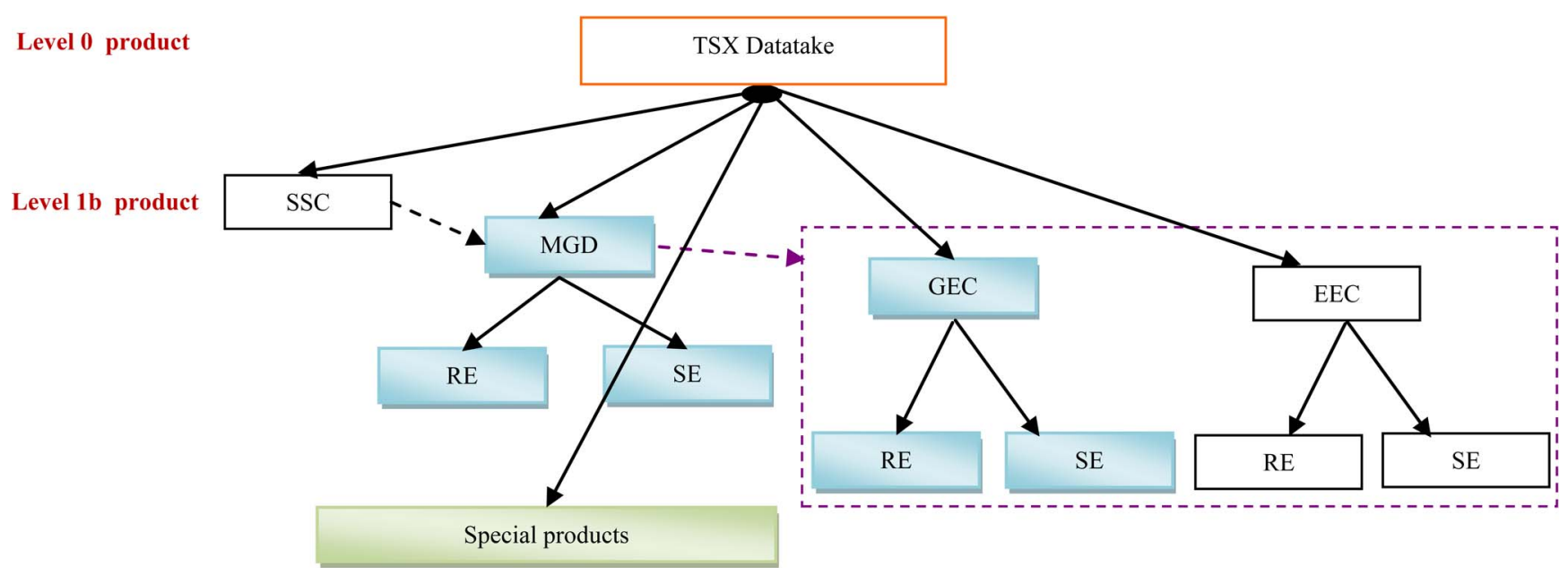

Fig. 7. TerraSAR-X product tree, where SSC is single look slant range, MGD is multilook ground range detected, GEC is geocoded ellipsoid corrected, and EEC is enhanced ellipsoid corrected [8]. The special MGD product is described in detail in [7] and presented in this section. The standard products are marked with blue color while the special process products are marked with green color.

The GEC product is a multilook detected product projected. The pixel localization accuracy is higher than in the case of MGD products.

The enhanced ellipsoid corrected (EEC) product is a multilook detected product. However, image distortions caused by varying terrain height are corrected using an external digital elevation model (DEM). The geometric accuracy of this product depends on the external DEM which usually is not delivered with the TerraSAR-X product.

The MGD, GEC, and EEC are TerraSAR-X detected products, while SSC is a TerraSAR-X complex product.

\section{B. Selected Products}

From all available TerraSAR-X basic L1b products, we focus only on detected products. However, the EEC product is discarded from our evaluation because an external DEM is needed and this product is more suitable for areas with higher deformation such as mountains.

The other two detected products are MGD and GEC. The MGD product is not affected by the interpolation and is more suitable for image processing and feature extraction than the geocoded products while the GEC product is georeferenced, and in some cases, this characteristic of the product is needed for a better location of the investigated area. Based on their characteristics, these two detected products [25] are a good option for applications such as indexing and recognition.

Regarding the product mode, we consider the SM mode because it is a standard mode available for all SAR satellites and the HS because it is the most used mode.

The geometric resolution configuration for the selected products is SE and RE.

\section{Methodology}

\section{A. Preprocessing}

For high-resolution images, pixel-based methods do not capture the contextual information (complex structures are usually a mixture of regions and cover many pixels; different distributions of the same objects can have different semantic meanings), and the global features describing the overall properties of the images are not accurate enough. Therefore, the general approach adopted in this paper is to divide the TerraSAR-X image into a number of patches (by tiling the product images), not overlapped, and to compute the feature extraction associated to each patch.

There are only few results in this direction [10]-[12] where the images were tiled into patches with different sizes. In [10], the patch size is $256 \times 256 \mathrm{~m}$ in order to ensure that the extracted information (features) captures the local characteristics within a patch rather the global features across the entire image.

In [11], the TerraSAR-X HS images with a resolution of about $1 \mathrm{~m}$ and the size of $10000 \times 10000 \mathrm{~m}$ were tiled into patches of $200 \times 200 \mathrm{~m}$ in order to characterize the large and relatively small structures available in the urban scene: Las Vegas (USA), Venice (Italy), Gizah (Egypt), and Gauting (Germany). From about 7000 patches, the proposed method allowed to extract about 30 different classes.

The authors of [12] proposed a patch contextual approach based on topographic independent component analysis for very high resolution satellite images. The method was tested with six monospectral QuickBird images. Each image was tiled into patches of $200 \times 200$ pixels, generating 20000 patches with their associated features that were used further to retrieve 18 classes.

In [22] and [23], the high-resolution SAR images acquired by the TerraSAR-X satellite over Stralsund (Germany) with the ground resolution of $6 \mathrm{~m} \times 6 \mathrm{~m}$ and over the Rosenheim (Germany) area with the ground resolution of $1.60 \mathrm{~m} \times 1.24 \mathrm{~m}$ are divided into hundreds of patches with overlapping. Some parts of the tiled patches may belong to many adjacent patches, and these patches might be assigned to different classes after the classification. They used a majority vote for these common parts of patches by distributing them to the most represented class in their neighborhood. 

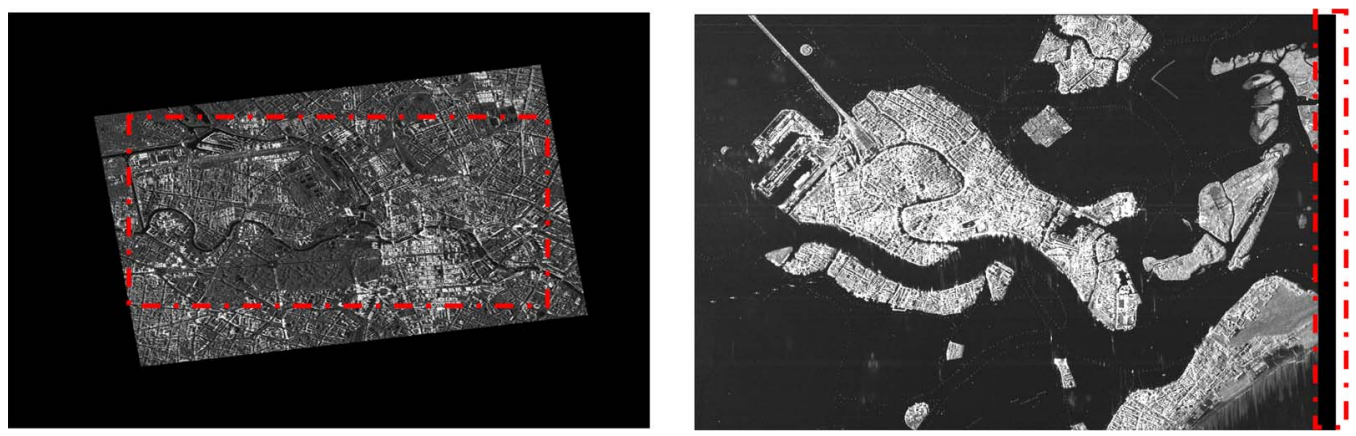

Fig. 8. Example showing (left side for Berlin) how to extract a rectangle from GEC product image (after which it is tiled into patches in step 2) and (right side for Venice) how to remove the black letter box from MGD product image before the tiling process.

Before the features are extracted, the data need to be preprocessed, and the following steps are applied.

1) Select a product from the data set: a) In the case of MGD products, no action is needed, and b) in case of GEC products, a rectangle is extracted from the product image (see the left side of Fig. 8) that contains only data and discards the rest of the image and the black letter box that appears in the image. The same region of interest (same rectangle) is extracted from the product image having the ascending and the descending looking necessary for comparisons in Section V-C.

2) Tile the product image (the full product image in the case of MGD products or the rectangle in the case of GEC products) into patches at different sizes in order to have the same area covered on ground. The size of the patch depends on the pixel spacing and resolution of each product, and both parameters can be found in the XML file of the TerraSAR-X product.

3) For the MGD products acquired until the end of 2012 (products generated with version 4.5 of TMSP), the tile procedure needs to take into account the reference numbers of columns and rows that appear in the image in order to remove the black letter box from the image (see the right side of Fig. 8) before tiling into patches. The black letter box can be in the right side of the product image when the acquisition is with an ascending looking and in the left side when the acquisition is with a descending looking. The parameters can be found in the XML file of each TerraSAR-X product.

4) Generate the quick looks (in jpg format without rescaling the data) of the tile patches needed for the visual management of the patches in the graphical user interface (GUI) (see Section III-C).

\section{B. PF Extraction Methods}

The following feature extraction methods are investigated and compared for TerraSAR-X products.

1) The GLCM is a second-order statistics of how often different combinations of pixel brightness values (gray levels) occur in an image [13].

The GLCM is created from a grayscale image by selecting either horizontal $\left(0^{\circ}\right)$, vertical $\left(90^{\circ}\right)$, or diagonal $\left(45^{\circ}\right.$ or $\left.135^{\circ}\right)$ orientation.

The size of GLCM depends on the number of gray values available in the image. For example, in [14], they obtain for an input image of 8 b, i.e., 256 values, a GLCM of $256 \times 256$ elements. In our case, we scale the radiometric range of the input images to 16 steps and obtain a GLCM size of $16 \times 16$ elements.

The features [15] computed from the GLCM are as follows: mean, variance, entropy, contrast, energy, correlation, homogeneity, autocorrelation, dissimilarity, cluster shade, cluster prominence, and maximum probability.

2) A GAF is a linear filter used in image processing. Frequency and orientation representations of a GAF are similar to those of the human visual system, and it has been found to be particularly appropriate for texture representation and discrimination. In the spatial domain, a 2-D GAF is a Gaussian kernel function modulated by a sinusoidal plane wave [16]. The GAFs are selfsimilar-all filters can be generated from one mother wavelet by dilation and rotation.

The implementation of the GAF convolves an image with a lattice of possibly overlapping banks of GAFs at different scales, orientations, and frequencies. The scale is the scale of the Gaussian used to compute the Gabor wavelet.

The features computed are mean and variance for different scales and orientations.

3) As proposed in [17], statistical features obtained from the filtered images using QMFs in synergy with some other features can be used for image (satellite image) indexing.

The number of features which can be obtained from the presented algorithm depends upon the level selected for the QMF subband decomposition like a wavelet. Features are nothing but the mean and variance of the four filtered and subsampled images in the QMF subband pyramid.

The texture parameters computed from the QMF banks are the mean and variance of the low-pass subband, horizontal subband, vertical subband, and diagonal subband.

4) The NSFT method for SAR image feature extraction and complex image information retrieval was first proposed in [18]. This nonparametric analysis is a form of time frequency analysis where the cutting of a spectrum allows the study of the phase responses of scatterers seen from different viewing angles.

The STFT extracts six nonlinear features: The first two features are based on statistical properties of the spectrum, and the next four features are timbre features used for music genre classification. 
The proposed algorithm is an implementation of the NSFT feature extraction, and the features computed are as follows: mean of the STFT coefficients, variance of the STFT coefficients, spectral centroid in range, spectral centroid in azimuth, spectral flux in range, and spectral flux in azimuth.

\section{Methodology}

The experiments reported in the literature [9]-[12] show that it is possible to detect a number of classes: In general, one obtains less than ten classes for low-resolution images and more than 30 classes for high-resolution images, depending on the bounds of classes which are defined by a user.

The general approach adopted here is to divide the TerraSAR-X image into a number of patches (by tiling the product image) and to compute the feature extraction associated to these patches.

To evaluate these issues, a tool based on support vector machine (SVM) [24] was built having as input the features computed in Section III-B. The SVM has been used for classification and learning in content-based image retrieval systems with very good performances. The kernel of this learning machine has the capability of performing very high accurate classification using a small number of examples for each class [26]. The relevance feedback (RF) software tool [20] supports users to search patches of interest in a large repository. The GUI of this tool allows human-machine interaction to rank the automatically suggested images which are expected to be grouped in the class of relevance. Visual supported ranking allows enhancing the quality of search results by giving positive and negative examples.

Using the SVM tool and the human expertise, we defined an annotated data set giving semantic meaning to the retrieved classes and group the patches accordingly. In our approach, the patches are assigned to only one class based on the dominant content in the patch (a patch is assigned to only one class).

For each evaluation, we tried to detect the classes among the number of patches/products of our data set. For each class, we give $20 \%$ of the patches for the training, and we try to detect the similar patches during 7-10 training iterations (this depends also by the class). The evaluations stop when the classified patches which are displayed by the SVM-RF tool remain in a stable result; this means that new patches are no longer retrieved from one iteration to another. The procedure is repeated 2-3 times for the same class, giving the same positive and negative examples in the same order, and after that, an average of the result is computed.

For the quantitative assessment, we compared the classification results with the annotated data set. Different metrics are proposed in the literature in order to evaluate the performance, and we considered the precision/recall metric for our evaluation.

The precision is defined as the fraction of the retrieved images which are relevant, while the recall is defined as the fraction of relevant images which have been retrieved

$$
\text { precision }=\frac{|A \cap B|}{|A|} \text { and } \text { recall }=\frac{|A \cap B|}{|B|}
$$

where $A$ represents the retrieved images and $B$ represents the relevant images.

In the first part, we propose to study and experimentally assess the most relevant $\mathrm{PF}$ behavior for indexing the content of TerraSAR-X images for which very few works are available in the literature. In the second part, we concentrate on the dependence of the orbit direction and incidence angle of TerraSAR-X products which was not investigated systematically until now.

The following issues will be analyzed in this paper:

1) use case 1: dependence on the resolution, pixel spacing, and PFs;

2) use case 2: dependence on the patch size, orbit looking, and incidence angle.

The evaluation methodology is common for both cases; maybe some small modifications can appear, but these are explained separately for each case.

\section{Use CASe 1: Dependence on the Resolution, PIXEL SPACING, AND PFs}

In this section, we analyze different TerraSAR-X products and propose the more appropriate one for classification purposes using the available data set.

In the first part of this section is presented the data set that is intended to be used in order to answer, in the second part of the section, the question regarding the influence of the SNR, pixel resolution, resolution, and PFs.

\section{A. Test Data Set}

In order to evaluate the performances of the PF algorithms in terms of SNR (related to resolution), pixel spacing, and resolution, a test data set is prepared.

Because the MGD products are more appropriated to feature extraction, the TerraSAR-X data set contains this type of product having both geometric resolution configurations (SE and RE). For SE and RE, the pixel spacing is lower than the resolution, and this means that we have an oversampling which affects the feature extraction algorithms.

The HS mode is chosen because it is the mode dedicated to the image processing of high-resolution data.

A special data set is generated, where a set of products with different levels of resolution and equivalent number of looks (ENL) was created from TMSP. It is important to mention that the idea behind this processing is that the pixel spacing and the resolution of the product are equal in order to have uncorrelated speckle. These products were generated directly from the TMSP, and the generation process is described [7] further in the next paragraphs.

The special MGD product is performed in the following way: oversampling and detection followed by low-pass filtering with inherent ground range projection and resampling. The transition from coherent complex data to incoherent intensity data comes along with a doubled extent of the azimuth and range spectra, respectively. Thus, prior to detection, the sampling of complex data is adequately increased in order to avoid aliasing of the detected data. The multilooking step within the TMSP is implemented as a time-domain convolution of incoherent image intensity values and a low-pass filter kernel. 
This is an alternative realization compared to the classical approach where individual looks are created as subbands in the spectral domain and transformed back to the time domain followed by incoherent look summation. The time-domain multilook filter serves as well as the interpolation kernel required for the SSC to MGD projection and resampling. One particular range and one particular azimuth filter are used for the processing of a given scene; thus, the number of looks is constant, while the ground range resolution varies to a small extent with range. This process allows a pyramid resolution with different ENLs and resolutions.

In the RE case, the goal is to obtain a higher ENL. In case of the SE variant, the best possible quadratic resolution is limited by the ground range resolution for step incidence angles below $32^{\circ}$. For the very step incidence angles, excessive azimuth bandwidth is turned into a slightly increased ENL. For incidence angles flatter than $32^{\circ}$, the resolution and ENL are almost constant on the order of $1.1 \mathrm{~m}$ and 1.0-1.2 ENL. In case of the RE variant, the resolution is limited to $4-3 \mathrm{~m}$. Thus, the achievable ENL is on the order of $5\left(\right.$ at $\left.20^{\circ}\right)$ and $8\left(\right.$ at $\left.45^{\circ}\right)$ [7].

The test data set-1 consists of five detected MGD products which are used to analyze the influence of the PFs at different resolutions. The sites cover Venice (Italy) and Toulouse (France), and the quick looks of these two areas are presented in Fig. 9(a) and (b). The multiresolution pyramid is shown in Fig. 9(c), where the special products at 1,2, and $4 \mathrm{~m}$ (products marked with a green color in Fig. 7) are in the left side and the standard products at $1 \mathrm{~m}$ and $2.9 \mathrm{~m}$ (products marked with a blue color in Fig. 7) are in the right side. A list of the parameters of each product available in test data set-1 from the multiresolution pyramid is presented in Table I.

The standard MGD products are SE and RE-HS image with single polarization $(\mathrm{HH})$ at a $1.15-\mathrm{m}$ resolution for $\mathrm{SE}$ and that at a 2.9-m resolution for $\mathrm{RE}$.

For SE, the pixel spacing is $0.5 \mathrm{~m}$, and the incidence angle is $38^{\circ}$ for Venice and $34^{\circ}$ for Toulouse. The size of the image is $10881 \times 15782$ pixels for Venice and $10568 \times 18319$ pixels for Toulouse.

For RE, the pixel spacing is $1.25 \mathrm{~m}$, and the incidence angles for Venice and Toulouse are the same with the ones for SE. The size of the image is $4353 \times 6313$ pixels for Venice and $4228 \times 7328$ pixels for Toulouse. The resolutions of the special products are 1-, 2-, and 4-m resolutions which are covering the resolution of the standard products. The mode of these products is HS mode with single polarization (HH).

\section{B. Dependence of the Resolution, Pixel Spacing, and PFs}

In order to perform an objective evaluation of the influence of the resolution, pixel spacing, and PFs, we compared these parameters for each product from the multiresolution pyramid.

Before using the test data set-1 for evaluation, a preprocessing step is necessary (see Section III-A). After the preprocessing is finished, the evaluation methodology presented in Section III-C is applied.

For the evaluation of the PFs, we proposed the following methods to be investigated and compared for TerraSAR-X products: GLCM, GAFs, QMFs, and NTFTs.

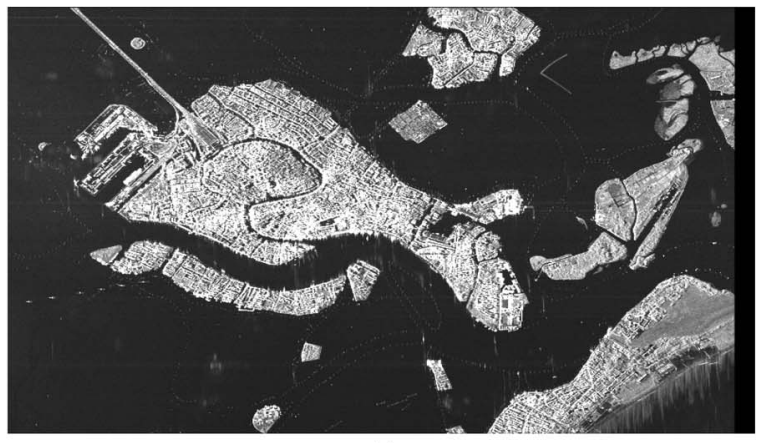

(a)

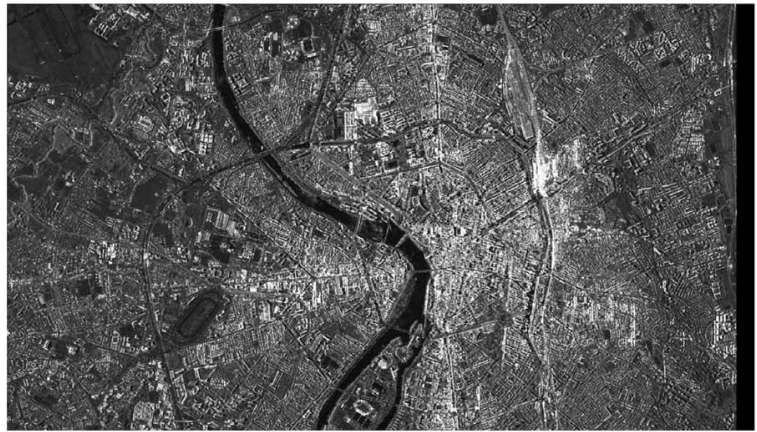

(b)

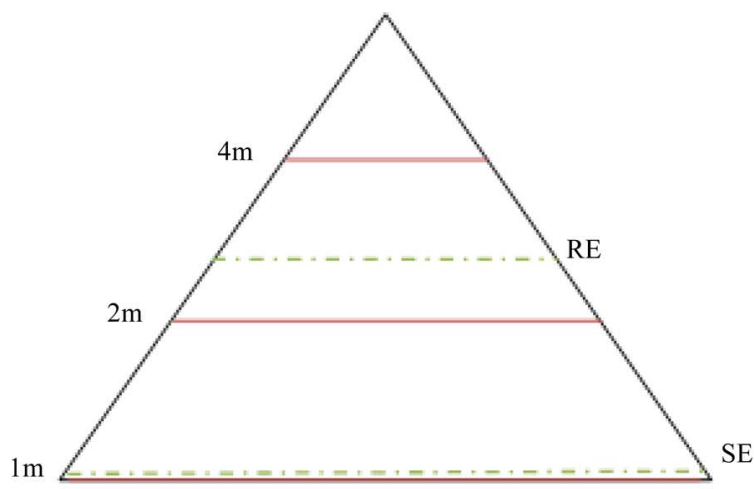

(c)

Fig. 9. Quick look of TerraSAR-X MGD-SE product with HS mode: (a) Venice (TSX1_SAR_MGD_SE_HS_S_SRA_20071124T165907_ 20071124T165908) and (b) Toulouse (TSX1_SAR_MGD_SE_HS_S_ SRA_20071127T174146_20071127T174147). (c) Multiresolution pyramid that corresponds to standard and special process product.

These four methods where chosen based on the following reasons.

1) GLCM is a very well-known method used to extract the texture features from an image.

2) GAFs is a linear filter used in image processing and a standard descriptor in MPEG 7 [27].

3) QMF is used intensively during the last two decades for image processing and speech signal processing [28], and the QMF banks are used to split a discrete-time signal into a number of bands in the frequency domain to process each subband in an independent manner.

4) NSFT is a method that is able to integrate the radiometric, the geometric, and texture properties of the SAR data [10].

The novelty of this investigation lies in the fact that these features are applied for SAR images (a summary of these is presented in Section II-C) and compared to each other for the 
TABLE I

Characteristics of TeSt DATA SET-1

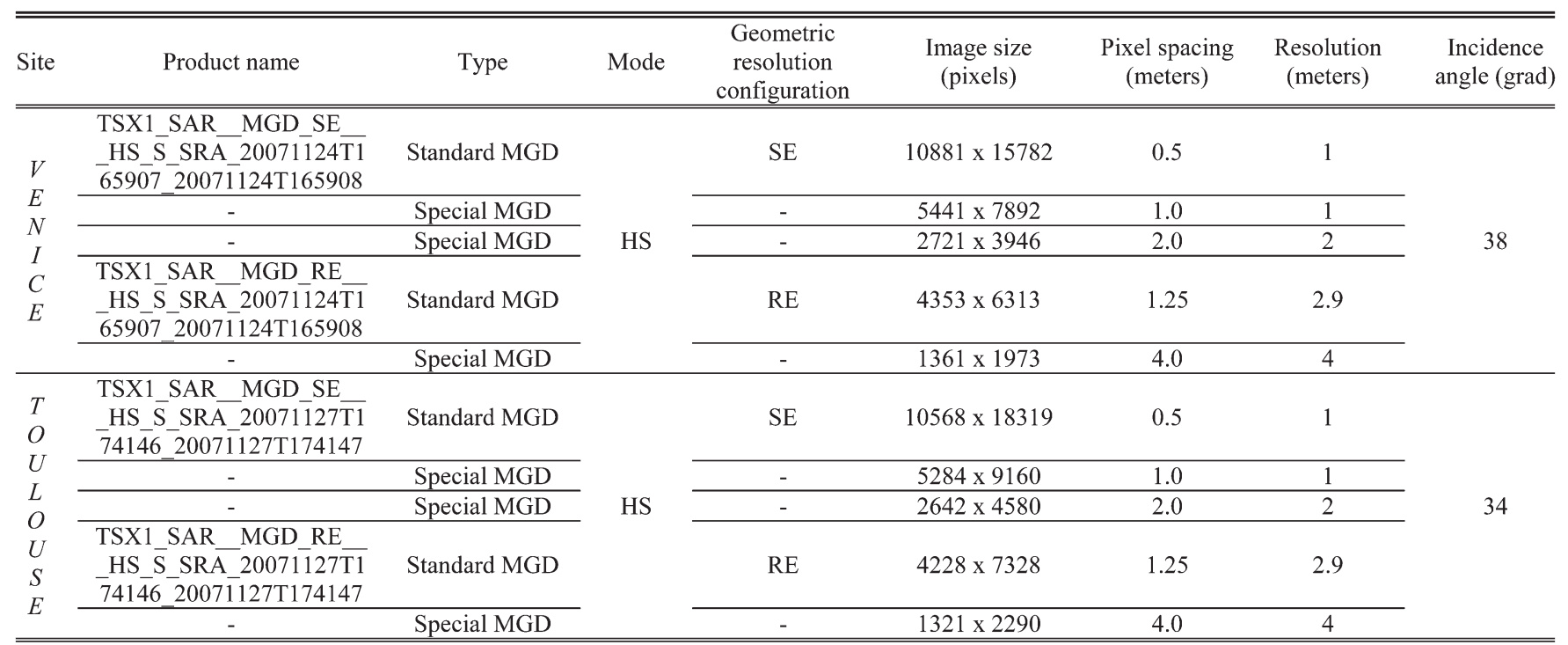
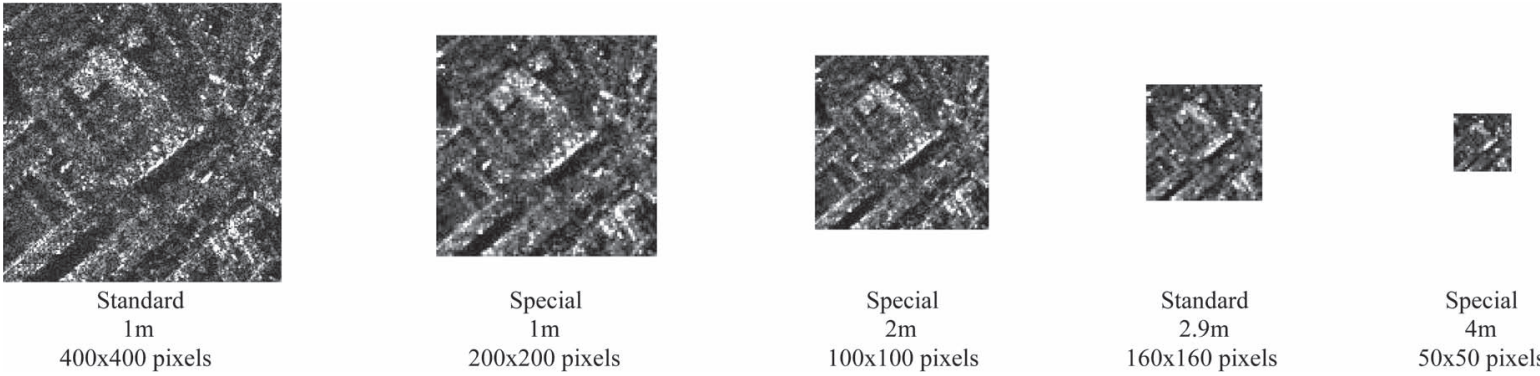

Fig. 10. Examples of patches extracted from the multiresolution pyramid over Toulouse site.

TerraSAR-X multiresolution pyramid (test data set-1). The first result of this evaluation was shortly presented in [20].

During the preprocessing, the product image is tiled into patches. The size of the patch is different from product to product in order to have the same area covered on the ground of about $200 \times 200 \mathrm{~m}$. This tiling depends on the reference number of rows and columns, pixel spacing, and resolution (available in the XML file of the TerraSAR-X product). The test data set- 1 after tiling has, in total, 2700 patches for each product (this means, in total, 13500 patches): 1026 for Venice and 1144 for Toulouse. The size of the patches for standard products is as follows: $400 \times 400$ pixels for MGD-SE and $160 \times 160$ pixels for MGD-RE. For special process products, it is as follows: $200 \times 200$ pixels for $1-\mathrm{m}$ resolution, $100 \times 100$ pixels for 2-m resolution, and $50 \times 50$ pixels for 4 -m resolution.

Fig. 10 shows the evolution of one patch (the quick looks of this patch) for all the products of the multiresolution pyramid. These examples are extracted from the data set available for the Toulouse area (site available in the test data set-1).

After the tiling, each patch has assigned a feature vector using one of the proposed algorithms. The feature vector for GLCM has a fix number of features for each orientation equal to 12 . In the evaluation, we considered all four orientations $\left(0^{\circ}, 45^{\circ}, 90^{\circ}\right.$, and $\left.135^{\circ}\right)$, and the feature vector has 48 values (4 orientations $\times 12$ features-GLCM_1_2_3_4). For GAFs, two versions are tested, the first with 2 scales and 2 orientations
(8 features-GAF 2_2) and the second with 4 scales and 6 orientations (48 features-GAF 4_6).

For QMFs, like for GAFs, two versions are selected, the first one with the number of levels equal to 1 (8 features-QMF 1) and the second one with the number of levels equal to 2 (14 features-QMF 2). For the last method, NSFT, the number of feature is fixed to 6 .

We defined a number of semantic classes and grouped the patches accordingly using the SVM-RF tool and the ground truth of Google Earth for the visual inspection. The list of all these classes is presented in Fig. 11, where one patch per class is shown with its semantic annotation (defined by visual inspection of the generated class) and the number of patches in each class.

For each feature extraction method, we tried to detect the classes among the number of identified patches of our data set by giving positive and negatives examples until the system/tool stays in a stable state. This means that new patches are no longer found from one iteration to another. After that, the metric (precision/recall) is computed for each feature, class, and product. This procedure is repeated for each product from the resolution pyramid, and the results are presented in the next figures.

First, we evaluate the classification performance of all PF algorithms for each class starting from the standard product and continue with the special process products. The retrieved results are compared using the annotated data set (containing the 


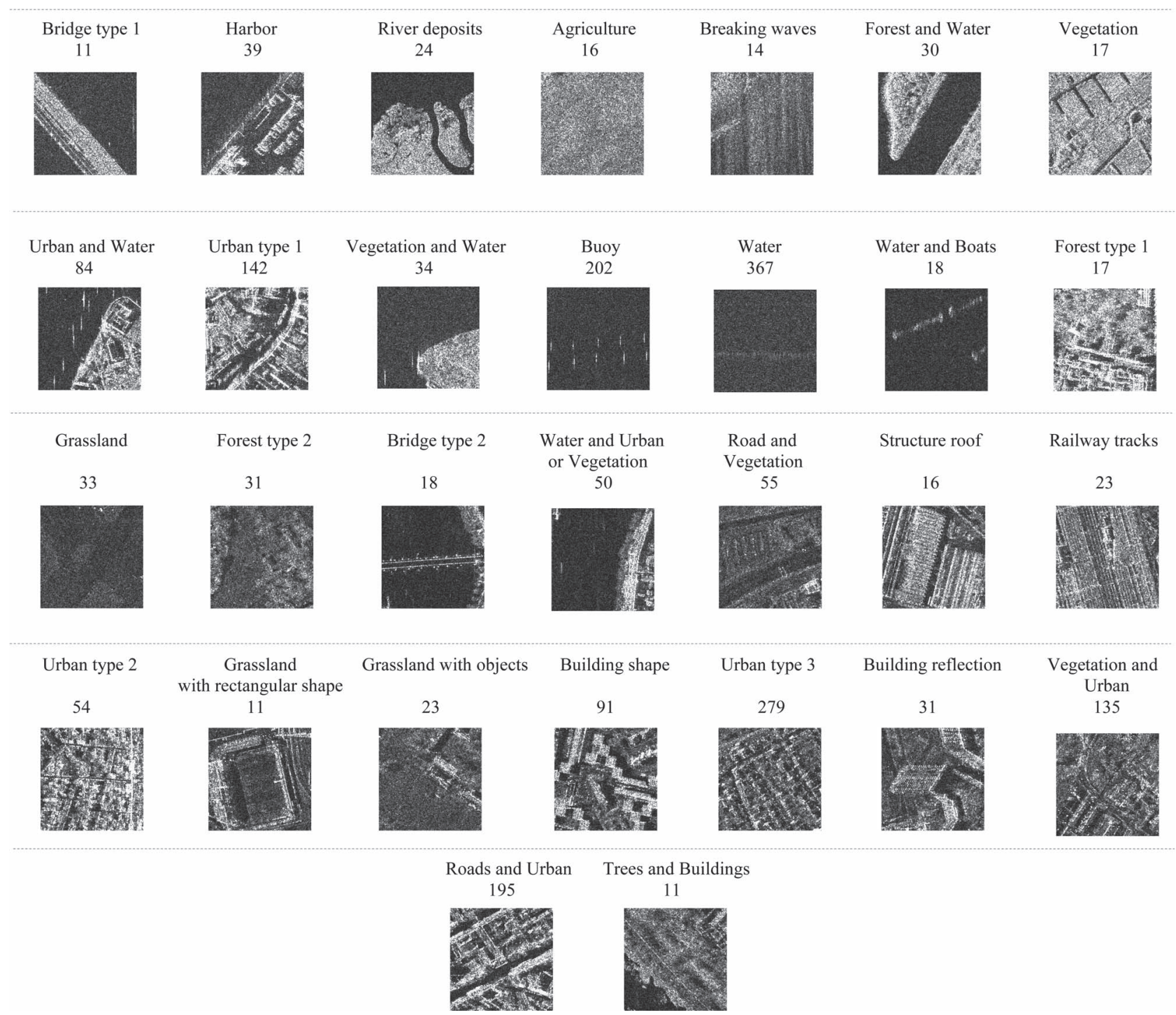

Fig. 11. Typical classes extracted from Venice and Toulouse (MGD product, HS mode, and patch size of $200 \times 200$ pixels).

30 classes presented with their semantic meaning in Fig. 11) for each product separately.

In Fig. 12, "per-class" classification accuracy is presented separately for each class and PF for the special MGD 1-m resolution product. With only few exceptions, the metric (precision) for each class is higher than $80 \%$ for almost all the PFs. Regarding the recall, the average for each class is between $30 \%$ and $42 \%$.

A similar representation can be presented as the one in Fig. 12 for all the products, but for a better understanding and comparison between features and product resolution, we decided to take the average over all the classes for each PF. The results (the precision/recall) are presented in Fig. 13 for each product and PF. Note that the QMF 2 is missing from this graph in case of the special product at $4 \mathrm{~m}$ because the size of the patch is too small to compute this type of feature. As can be seen in Fig. 13, in most cases, the first two values of the recall correspond to GAFs and QMFs. However, for precision, the results are divided between GAFs, QMFs, and GLCM.
In Fig. 14, the best performances are presented as a synthesis, where each value is an average computed over all classes and PFs of each product.

\section{Conclusion}

After the investigation and comparison between the PFs, classes, and products, the following conclusions can be drawn.

1) The GAFs with different scales and orientations perform better than the other features, particularly when the $r e$ call is computed. The second performance in recall is obtained for QMFs. The QMF has the advantage of being faster (in required run time for feature computation) than the GAFs. In the case of precision, one of the following algorithms, GAFs (with different scales and orientations), QMFs (with different levels of the wavelet decomposition), or GLCM (with all four orientations), can be taken into account for classification.

2) In the synthesis figure (see Fig. 14), we can notice that the special MGD-SE product at 4-m resolution performs 

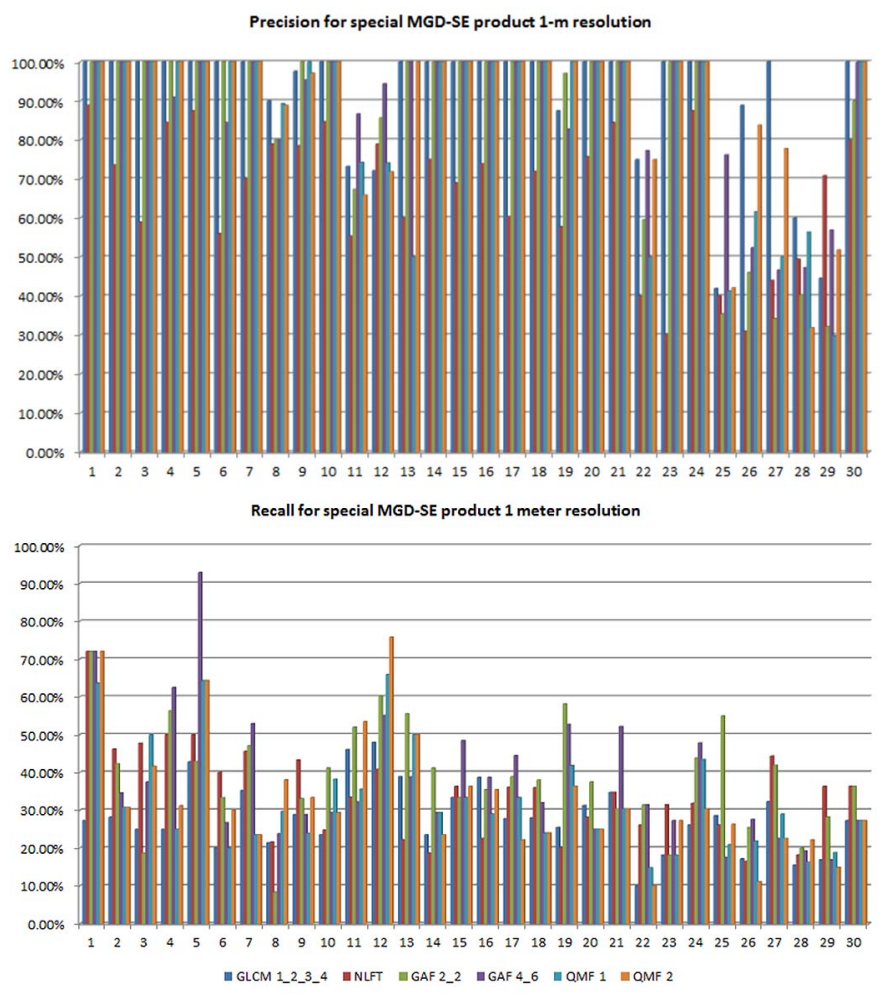

Fig. 12. Per-class classification accuracies (precision-top of this figure; recall-bottom of this figure) of all proposed feature extraction algorithms: GLCM (all 4 orientations), NLFT, GAFs (with 2 scales and 2 orientations and with 4 scales and 6 orientations), and QMFs (with number of levels equal to 1 and 2). The plot results are for Venice and Toulouse in the case of special MGD 1 -m resolution product with a patch size of $200 \times 200$ pixels.

better in precision than the rest of the products. In terms of recall, the standard MGD-RE product is the better one. We consider that recall is more important than precision because our intention is to find relevant patches into a data set of hundreds of products, which means millions of patches.

3) Comparing the results between the standard MGD-SE product and the special product at $1 \mathrm{~m}$, the performance of the product with the pixel spacing equal to the resolution is better than the other one. In terms of the other standard product, MGD-RE, the precision is between the special products at 2 and $4 \mathrm{~m}$, and for recall, the standard product is better than the special products.

4) Starting from the previous observation, we try to improve the performances of the standard MGD-RE product in precision by subsampling the data. For this, the best two PFs in recall (these two PFs corresponds to GAF 4_6 and GAF 2_2; see Fig. 12) are selected, and then, the standard MGD-RE product is subsampled. After the features are computed and the metric is calculated, the result of the subsampled data in precision is improved by $3 \%$ in the case of GAF $2 \_2$ and by $0.5 \%$ in the case of GAF 4_6, but overall, the precision for GAF 4_6 is higher by $10 \%$ than that of the GAF 2_2. Another advantage of subsampling the data is that the time needed to extract the features is smaller because the features are computed on data two times smaller.
Based on the presented results, we recommend the standard product, the RE, and the GAFs with different scales and orientations and/or the QMFs with different numbers of levels as PFs.

\section{Use Case 2: Dependence on the Patch Size, Orbit Direction, AND InCIDENCE ANGLE}

The results obtained in the previous section (using standard product, RE, with single polarization, and GAFs and QMFs as $\mathrm{PFs}$ ) are considered as a basis for building the new data set.

In this section, we evaluate, aside from the optimal patch size (need for feature extraction), the orbit direction and incidence angle.

In order to have a correct evaluation of the orbit looking and incidence angle, our data need to answer to three requirements: 1) to be acquired with the ascending and the descending looking; 2) to have two-three incidence angles covering the minmax range of the sensor with the same orbit looking (ascending or descending); and 3) to cover the same area in each case.

Because the previous sites (Venice and Toulouse) used for evaluation in "use case 1" were not available on EOWEB portal (from where the TerraSAR-X products can be downloaded for scientific purpose) with our specific requirements regarding the orbit direction and incidence angle, we selected another product and area. This detected product is GEC georeferenced, and the area covers Berlin and Ottawa.

After the test data set is built in Section V-A, the optimal patch size is defined in Section V-B while Sections V-C and V-D evaluate the dependence of the product image according to various parameters (e.g., orbit direction, incidence angle, etc.). The test data set is preprocessed (see Section III-A) before being used for evaluation. For evaluation, the same methodology is applied and presented in Section III-C.

\section{A. Test Data Set}

The test data set-2 consists in GEC products radiometrically enhanced (GEC-RE) covering the area of Berlin (Germany) and Ottawa (Canada). The product modes are SM and HS with single polarization $(\mathrm{HH})$. The characteristics of these products are presented in Table II.

In case of the SM mode, the products cover the Berlin area (see Fig. 15). The resolution of the product is $6.5 \mathrm{~m}$ for the ascending looking with $29^{\circ}$ for the incidence angle and $7.8 \mathrm{~m}$ for the descending looking with $37^{\circ}$ for the incidence angle. For this GEC-RE product, the pixel spacing is different, $2.75 \mathrm{~m}$ for the ascending looking and $2.5 \mathrm{~m}$ for the descending looking. The size of the image for the ascending and the descending looking is $6419 \times 4620$ pixels and $7060 \times 5081$ pixels, respectively.

In the case of the HS mode, the GEC-RE products cover the Berlin (a small part of the city is common with the one from $\mathrm{SM}$ ) and Ottawa area. The pixel spacing is $1.25 \mathrm{~m}$, and the resolution is $2.9 \mathrm{~m}$ for both sites.

For each site, two products are considered having the incidence angle closer to the lower and the upper bound of the sensor range. In the case of Berlin (see Fig. 15), these values are $30^{\circ}$ and $42^{\circ}$, and in the case of Ottawa, these are $27^{\circ}$ and 

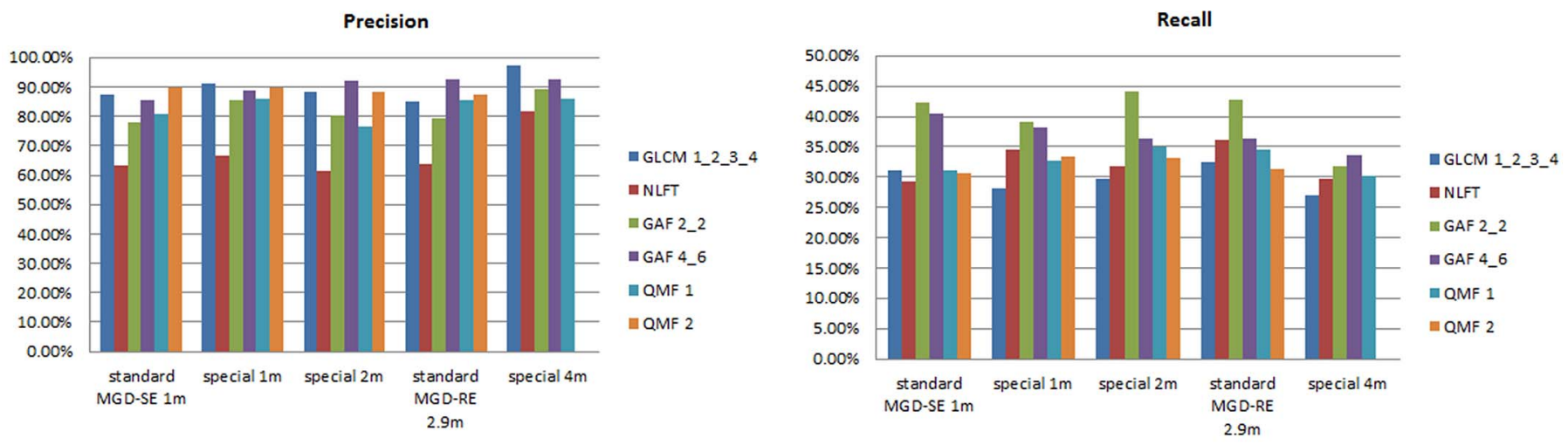

Fig. 13. Per-feature classification accuracies as average over all 30 retrieved classes available in test data set-1 for Venice and Toulouse.

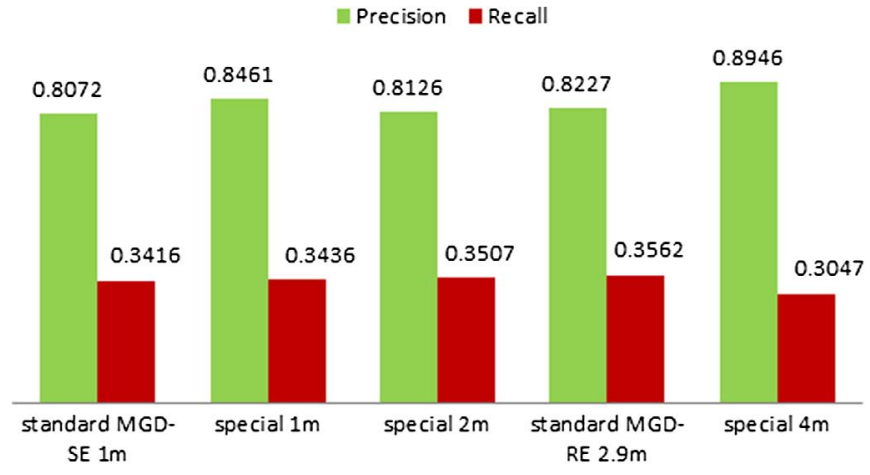

Fig. 14. Per-product classification accuracies as average over all algorithms and classes available in the test data set-1.

$41^{\circ}$. The size of the selected images is $5549 \times 3368$ pixels with an ascending looking for Berlin and $4783 \times 3381$ pixels with a descending looking for Ottawa.

\section{B. Dependence of the Patch Size (Analyzing Window)}

We study the optimal patch size (analyzing window) because of three reasons.

1) If the structure inside the patch is homogeneous/stationary, we assume that the variation/modification of the features can be improved by modifying the dimension of the patch.

2) If the structure inside the patch is heterogeneous, the increasing size of the patch can be a solution, but a size too large can lead to confusion. In this case, more objects can be embedded in the same patch.

3) If the size of the patch is too small, the objects can be truncated.

In the literature, the size of the analyzing window (patch size) is chosen so that the patch can cover an entire object on ground. This value of the patch is around $200 \times 200 \mathrm{~m}$ and needs to take into account the resolution of the product (see Fig. 16).

Another limitation of the patch size can come out when the feature extraction algorithms are applied if the size is too small (see Fig. 12 in the case of the QMF 2 algorithm).

Tests are carried for TerraSAR-X geocoded products (GEC) with SM and HS modes on Berlin because of the highest diversity in content of the site. For each product and mode, we select two analyzing windows (patch sizes) adapted to the resolution, and the precision/recall is computed for both $\mathrm{PF}$ algorithms (GAFs and QMFs).

The results of the investigation will be as follows: 1) the optimal analyzing window size (patch size) for the TerraSAR-X product with SM and HS modes, radiometically enhanced available in the test data set-2 and 2) the best PF algorithm in order to have this optimal window.

The methodology is the one presented in Section III-C. With a change after the tiling step, an intermediate step is applied in order to subsample the data (e.g., patches). This step is introduced based on the fourth conclusion derived from "use case 1."

In order to correctly tile the product image (TerraSAR-X SM mode with RE) into patches and to have the same covered area on the ground, we need to take into account the pixel spacing and resolution.

The first step is to set the patch size for the GEC-SE product and, after that, to compute the patch size for the GEC-RE product. The second step is to calculate the pixel spacing factor factor $=$ pixel spacing $-G E C \_R E /$ pixel spacing $G E C \_S E$ that is necessary for finding other values of the patch; for the ascending looking, the factor is 2.2 , and for the descending looking, it is 2 .

After this factor is calculated, two patch sizes for the GEC-SE product with an ascending looking are set equal to $220 \times 220$ pixels and $176 \times 176$ pixels. The patch sizes of the GEC-RE product is equal to (patch_size $-G E C \_R E=$ patch_size-GEC_SE/factor) $100 \times 100$ pixels and $80 \times 80$ pixels, respectively. For the GEC-RE product with HS, the analyzing windows are the same with the ones for the GEC-SE product because the pixel spacing and resolution of the two products are the same (e.g., $1.25 \mathrm{~m}$ for pixel spacing and $2.9 \mathrm{~m}$ for resolution). The patch is subsampled before being used for feature extraction.

In the case of GEC-RE products with the SM mode, the data set has 5555 patches (covering the Berlin city and the eastern suburbs of the city) after tiling: 2409 patches are with the size of $50 \times 50$ pixels, and 3546 patches are with the size of $40 \times$ 40 pixels.

For the HS mode of the same type of product, the number of patches is equal to 365 with the size of $110 \times 110$ pixels and 503 with the size of $88 \times 88$ pixels covering only a part of the city of Berlin. 
TABLE II

Characteristics of TeSt DATA SET-2

\begin{tabular}{|c|c|c|c|c|c|c|c|c|}
\hline Site & Product name & $\begin{array}{l}\text { Type / } \\
\text { Mode }\end{array}$ & $\begin{array}{l}\text { Geometric } \\
\text { resolution } \\
\text { configuration }\end{array}$ & $\begin{array}{l}\text { Image } \\
\text { size } \\
\text { (pixels) }\end{array}$ & $\begin{array}{l}\text { Pixel spacing } \\
\text { (meters) }\end{array}$ & $\begin{array}{l}\text { Resolution } \\
\text { (meters) }\end{array}$ & $\begin{array}{l}\text { Incidence } \\
\text { angle (grad) }\end{array}$ & $\begin{array}{c}\text { Orbit } \\
\text { looking }\end{array}$ \\
\hline \multirow{4}{*}{$\begin{array}{l}B \\
E \\
R \\
L \\
I \\
N\end{array}$} & $\begin{array}{c}\text { TSX1_SAR_GEC_RE } \\
\text { SM_S_SRA_20100628T164 } \\
400 \text { 20100628T164403 }\end{array}$ & \multirow{2}{*}{$\begin{array}{c}\text { GEC } \\
\text { SM }\end{array}$} & \multirow{2}{*}{ RE } & $6419 \times 4620$ & 2.75 & 6.5 & 29 & ascending \\
\hline & $\begin{array}{l}\text { TSX1_SAR_GEC_RE } \\
\text { SM_S_SRA_20100630T052 } \\
\text { 522_20100630T052525 }\end{array}$ & & & $7060 \times 5081$ & 2.5 & 7.8 & 37 & descending \\
\hline & $\begin{array}{c}\text { TSX1_SAR_GEC_RE } \\
\text { HS_S_SRA_20090323T164 } \\
\text { 352_20090323T164352 }\end{array}$ & \multirow{2}{*}{$\begin{array}{c}\text { GEC } \\
\text { HS }\end{array}$} & \multirow{2}{*}{$\mathrm{RE}$} & $5549 \times 3368$ & 1.25 & 2.9 & 30 & ascending \\
\hline & $\begin{array}{l}\text { TSX1_SAR_GEC_RE } \\
\text { HS_S_SRA_20091103T165 } \\
\text { 235_20091103T165236 }\end{array}$ & & & $5549 \times 3368$ & 1.25 & 2.9 & 42 & ascending \\
\hline $\begin{array}{l}O \\
T \\
T\end{array}$ & $\begin{array}{l}\text { TSX1_SAR_GEC_RE } \\
\text { HS_S_SRA_20110727T112 } \\
052 \text { 20110727T112052 }\end{array}$ & \multirow{2}{*}{$\begin{array}{c}\text { GEC } \\
\text { HS }\end{array}$} & \multirow{2}{*}{$\mathrm{RE}$} & $4783 \times 3381$ & 1.25 & 2.9 & 27 & descending \\
\hline $\begin{array}{l}A \\
W \\
A\end{array}$ & $\begin{array}{l}\text { TSX1_SAR_GEC_RE } \\
\text { HS_S_SRA_20110802T111 } \\
219 \text { 20110802T111220 }\end{array}$ & & & $4783 \times 3381$ & 1.25 & 2.9 & 41 & descending \\
\hline
\end{tabular}
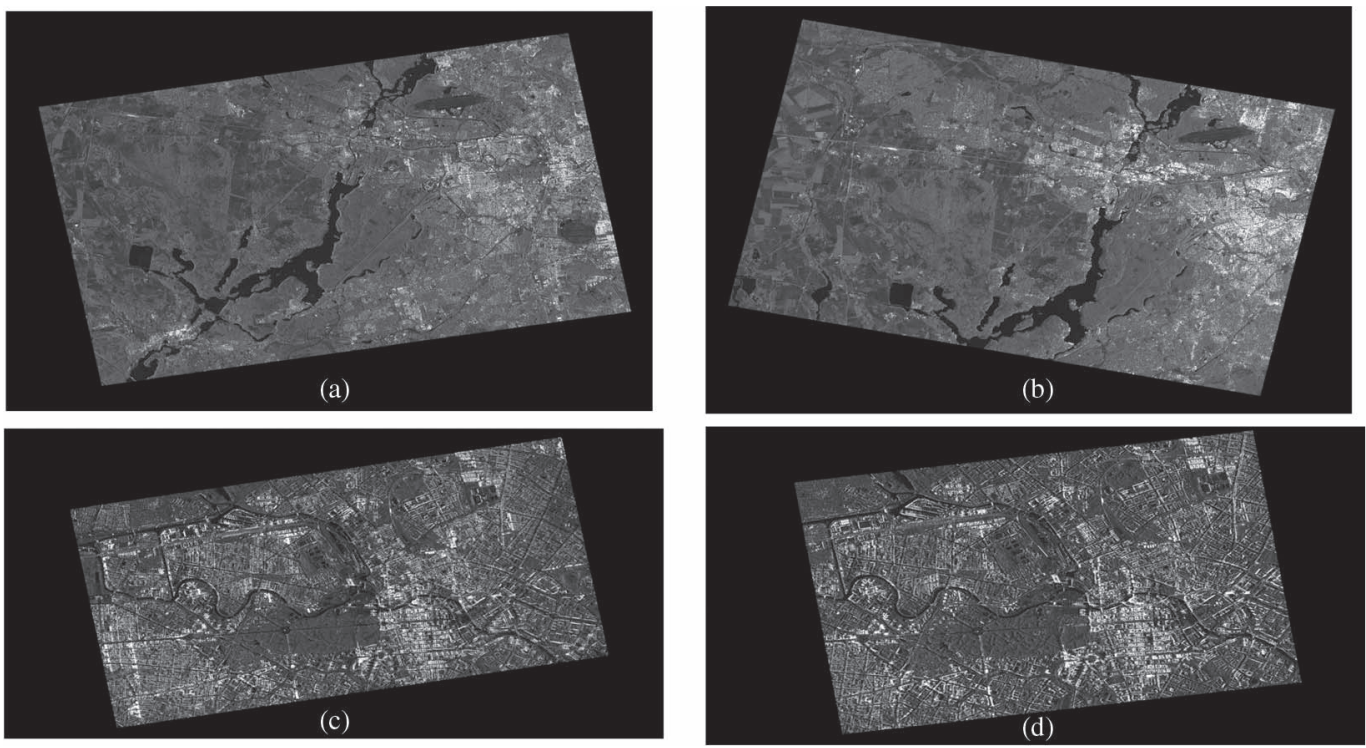

Fig. 15. (Top of the figure) Quick look of TerraSAR-X GEC-RE product with SM mode and with (a) ascending looking and (b) descending looking covering the Berlin area. (Bottom of the figure) Quick look of TerraSAR-X GEC-RE product with HS mode and with (c) incidence angle equal to $30^{\circ}$ and (d) incidence angle equal to $42^{\circ}$ covering a part of Berlin area.

For this data set, a number of semantic classes are defined, and the patches are grouped accordingly, using the ground truth of Google Earth. The list of all these classes (17 classes for SM mode and 11 classes for HS mode) is presented in Figs. 17 and 18 , where one patch per class is shown with the associated semantic meaning and the number of patches in each class.

GAFs (GAFs with 4 scales and 6 orientations) and QMFs (QMFs with the decomposition wavelet equal to 1) are applied as PFs to the subsampled data.

In the next two figures (see Figs. 19 and 20), the "per-class and patch size" classification accuracy over GAF and QMF algorithms of the SM and HS products is presented. In Fig. 19, the results of the precision/recall for the GEC-RE product with the SM mode are shown for two patch sizes, $50 \times 50$ pixels and $40 \times 40$ pixels. The two PFs are investigated, and as can be seen in precision, the results are better for GAFs, contrary to

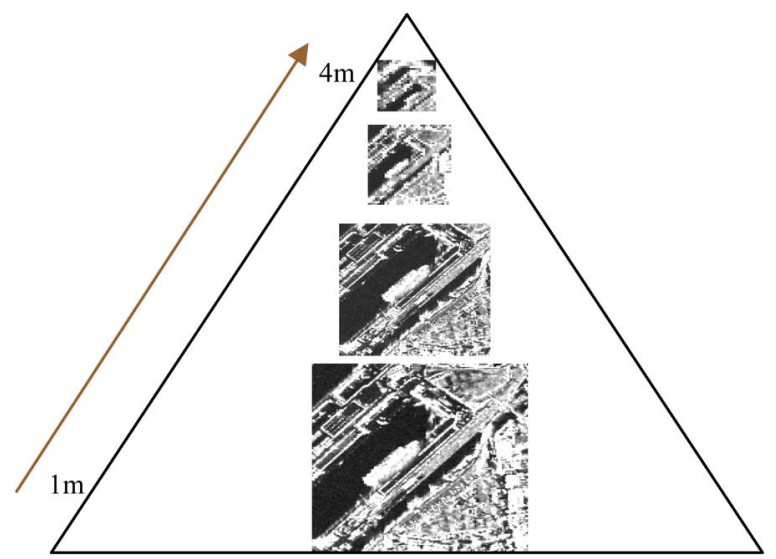

Fig. 16. Example of four patches (the port area in Venice) having the resolution from 1 to $4 \mathrm{~m}$. 


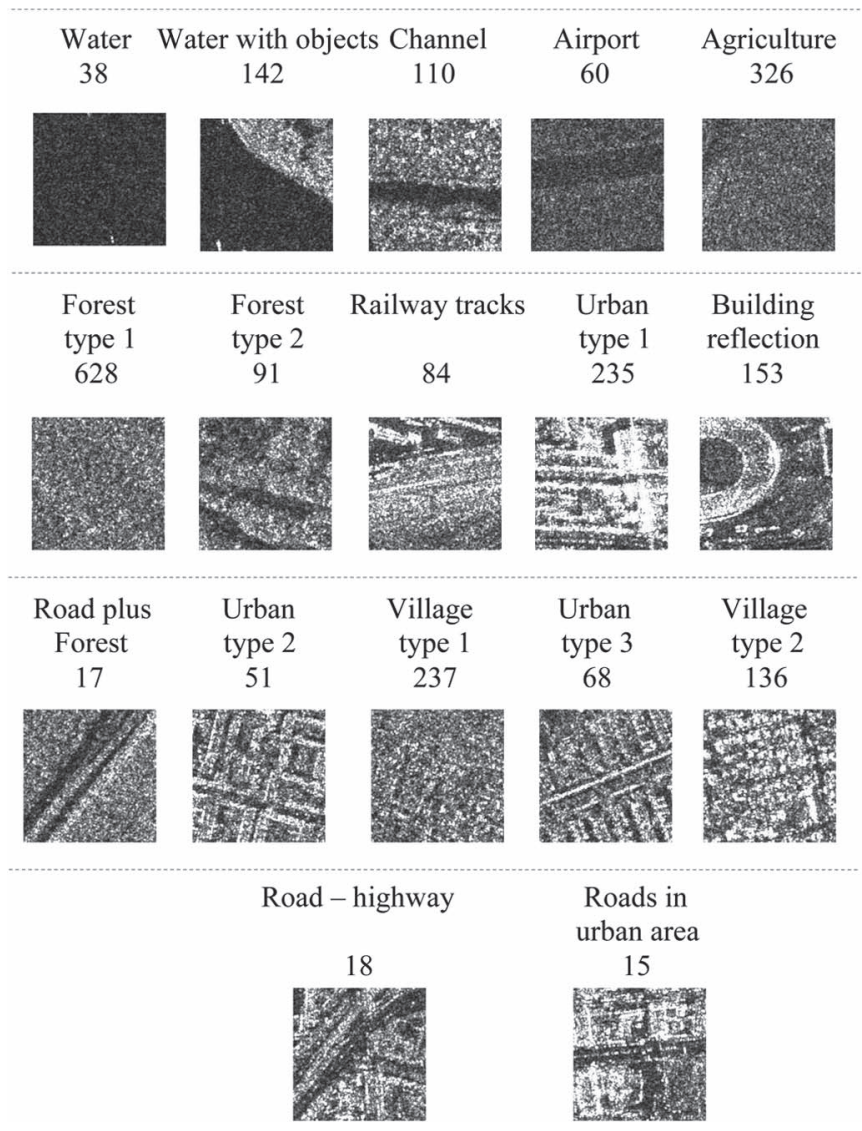

Fig. 17. Typical classes (patch size of $50 \times 50$ ) extracted from Berlin image (TerraSAR-X GEC-RE product, with SM mode).

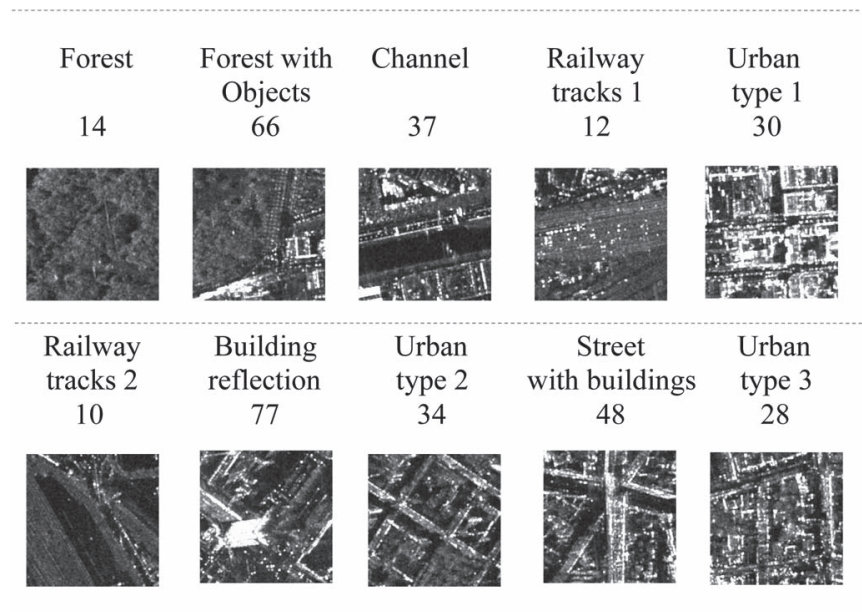

Sport and other fields

9

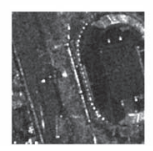

Fig. 18. Examples of classes (patch size of $110 \times 110$ ) extracted from Berlin image (TerraSAR-X GEC-RE product, with HS mode).

recall where the QMF algorithm is better. This remark is valid for both analyzing windows (patch sizes) and the majority of the classes.

Taking the average over all the classes and PFs (see the first two bars of the graph in the left side of Fig. 21), the product which is tiled at $40 \times 40$ pixels has higher classification accuracy. The accuracy average (precision/recall) is $68.22 \%$ and $27.91 \%$, respectively, for a patch tiled at $50 \times 50$ pixels and $78.92 \%$ and $28.69 \%$, respectively, for the second size of the patch $(40 \times 40$ pixels $)$.

For the second evaluation, the accuracy of the PF for each class in the case of the GEC-RE product with the HS mode is presented in Fig. 20. Better performances in classification are obtained when the image is tiled at $110 \times 110$ pixels than at $88 \times 88$ pixels.

The accuracy classification performances for SM and HS modes and for each patch size, where each value of the precision/recall is an average computed over all classes and PFs, are presented in Fig. 21.

Based on the previous results regarding the PFs, patch size, and geocoded products with SM and HS modes, the following conclusions can be drawn.

1) The GAFs perform better than the other features when the precision is computed. Regarding the recall, the better performances are obtained for QMFs.

2) The optimal patch size is depending on the product mode and the resolution of this (see Fig. 21). Comparing the patch size separately for each product, the optimal analyzing window (patch size) is as follows: $40 \times 40$ pixels for the GEC-RE product with the SM mode and $110 \times 110$ pixels for the GEC-RE product with the HS mode. All these patches are subsampled before the feature algorithms are applied.

3) The classification accuracy (precision/recall) is higher for the HS mode than for the SM mode.

\section{Dependence of the SAR Imaging Orbit Looking}

This evaluation is very important for indexing large TerraSAR-X data sets that have a higher variability of the orbit looking. The images and signature of human man-made structures are sensitive to the orbit direction.

The experiments run in order to identify the dependence of the SAR imaging with orbit looking are done using the optimal TerraSAR-X product, patch size, and the best two PFs found in the previous sections.

Because, for this investigation, the acquired data must have the ascending and the descending looking for the same area, the only product available in the TerraSAR-X archive (without any new acquisition of the satellite) was the GEC product with the SM mode. For this investigation, we select for our test data set-2 an urban area that corresponds to Berlin (Germany) for which the acquisition has been done with the ascending and the descending looking.

In order to understand the difference between ascending looking and descending looking, in Fig. 22, four examples are presented containing man-made structure and airplane tracks.

The methodology of the evaluation is the one presented in Section III-C, and it is applied after the preprocessing stage is completed (see Section III-A).

Taking the best PFs GAFs (with 4 scales and 6 orientations) and QMFs (with a level decomposition equal to 1) and the patch size for the GEC-RE product with the SM mode and with 
Precision for GEC-RE product with SM mode and with patch size of $50 \times 50$ pixels
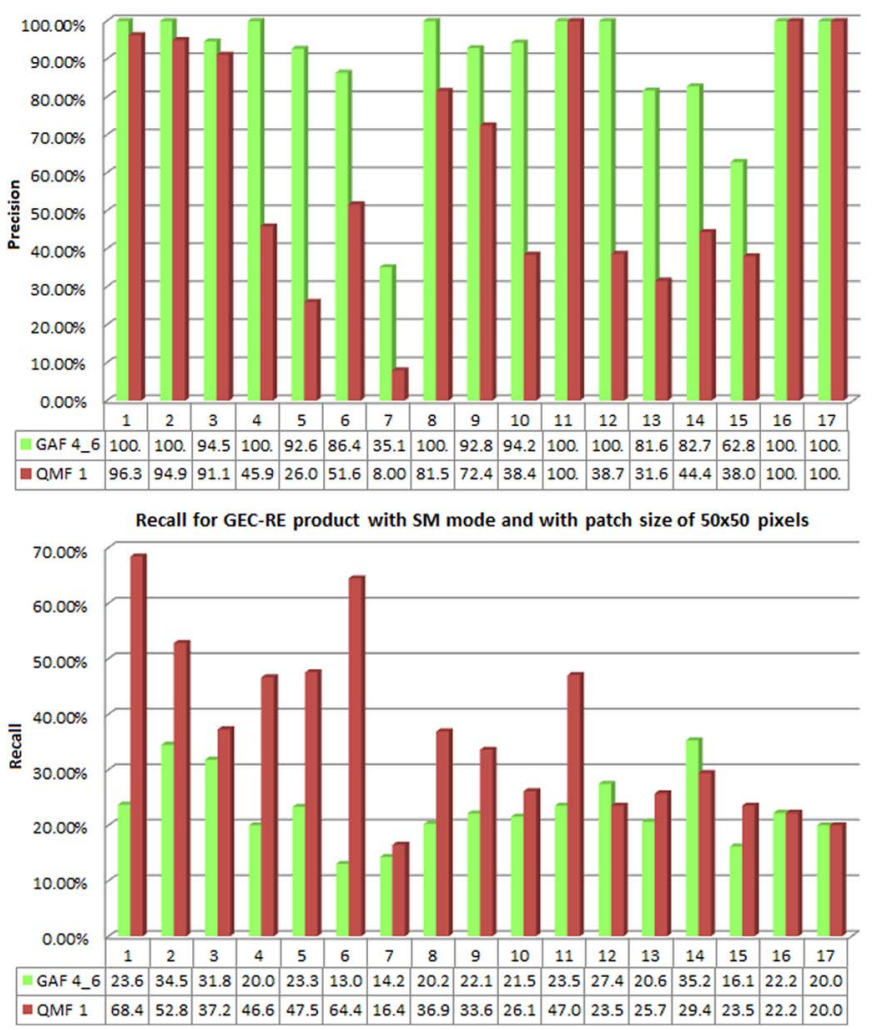

Precision for GEC-RE product with SM mode and with patch size of $40 \times 40$ pixels

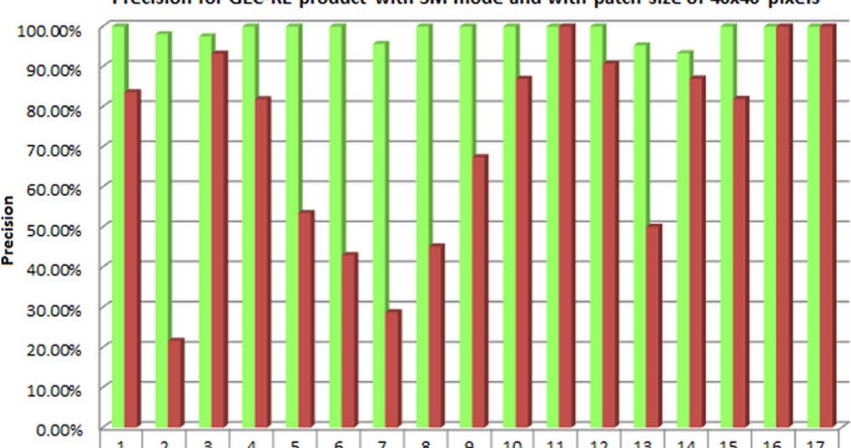

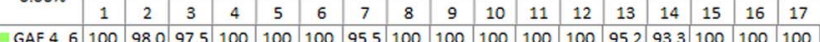

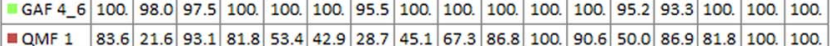

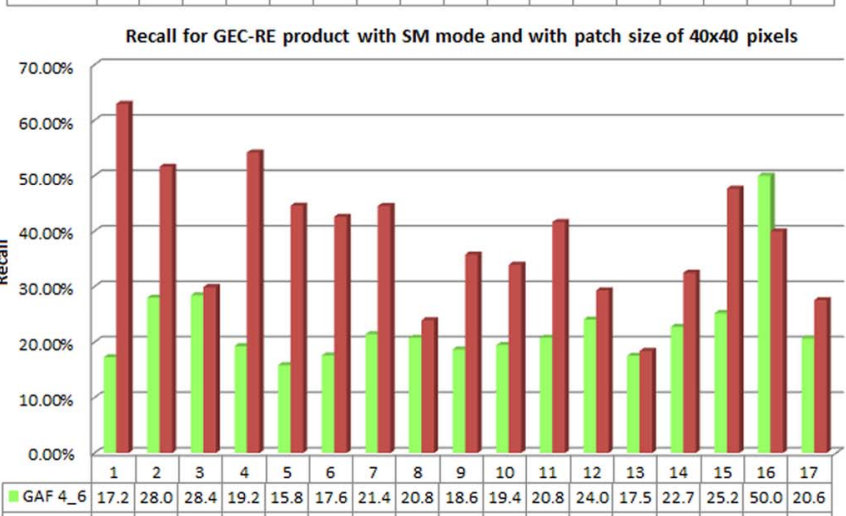

\begin{tabular}{|l|l|l|l|l|l|l|l|l|l|l|l|l|l|l|l|l|l|}
\hline GAF 4_6 & 17.2 & 28.0 & 28.4 & 19.2 & 15.8 & 17.6 & 21.4 & 20.8 & 18.6 & 19.4 & 20.8 & 24.0 & 17.5 & 22.7 & 25.2 & 50.0 & 20.6 \\
\hline M QMF 1 & 62.9 & 51.6 & 29.9 & 54.2 & 44.6 & 42.6 & 44.5 & 23.9 & 35.8 & 33.9 & 41.6 & 29.3 & 18.4 & 32.5 & 47.7 & 40.0 & 27.5
\end{tabular}

Fig. 19. Per-class classification accuracies (precision-top of the figure; recall-bottom of the figure) of the GAFs and QMFs for Berlin in the case of the GEC-RE product with SM mode and with patch sizes of (in the left) $50 \times 50$ pixels and (in the right) $40 \times 40$ pixels.

Precision for GEC-RE product with HS mode and with patch size of 110x110 pixels

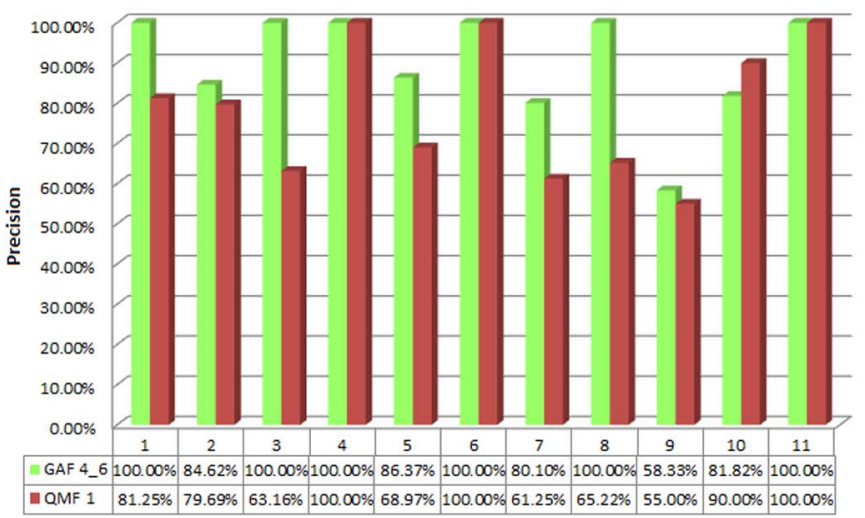

Recall for GEC-RE product with HS mode and with patch size of 110x110 pixels

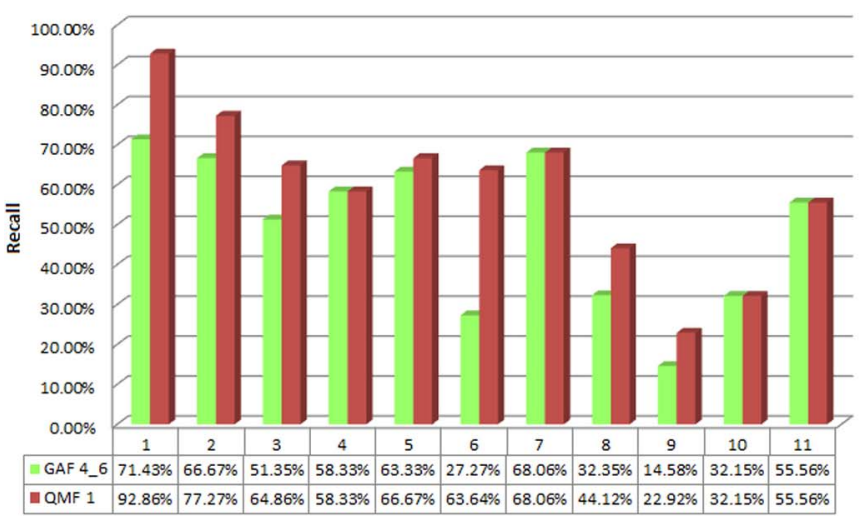

Precision for GEC-RE product with HS mode and with patch size of $88 \times 88$ pixels

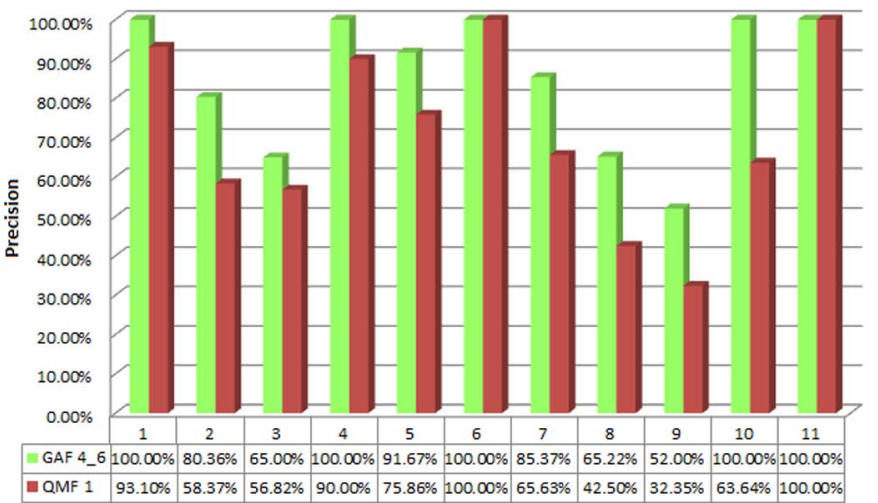

Recall for GEC-RE product with HS mode and patch size of $88 \times 88$ pixels

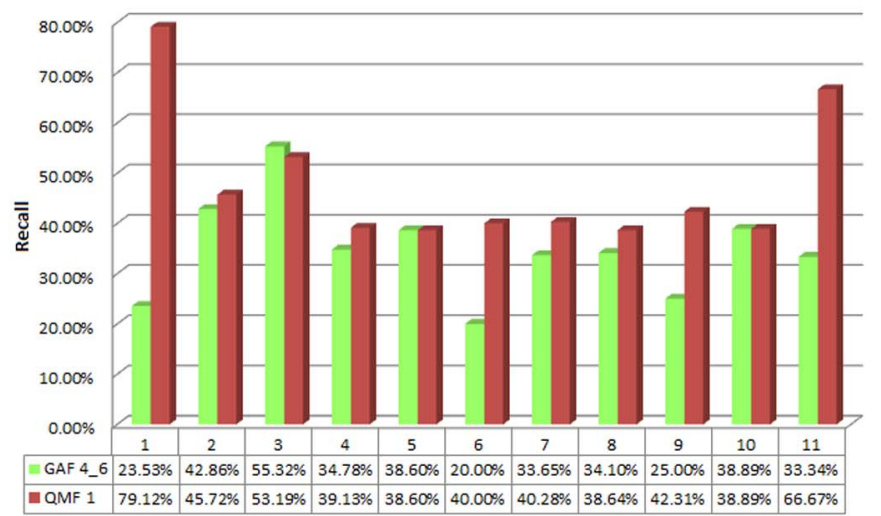

Fig. 20. Per-class classification accuracies (precision-top of the figure; recall—bottom of the figure) of the GAFs and QMFs for Berlin in the case of GEC-RE product with HS mode and with patch sizes of (in the left) $110 \times 110$ pixels and (in the right) $88 \times 88$ pixels. 


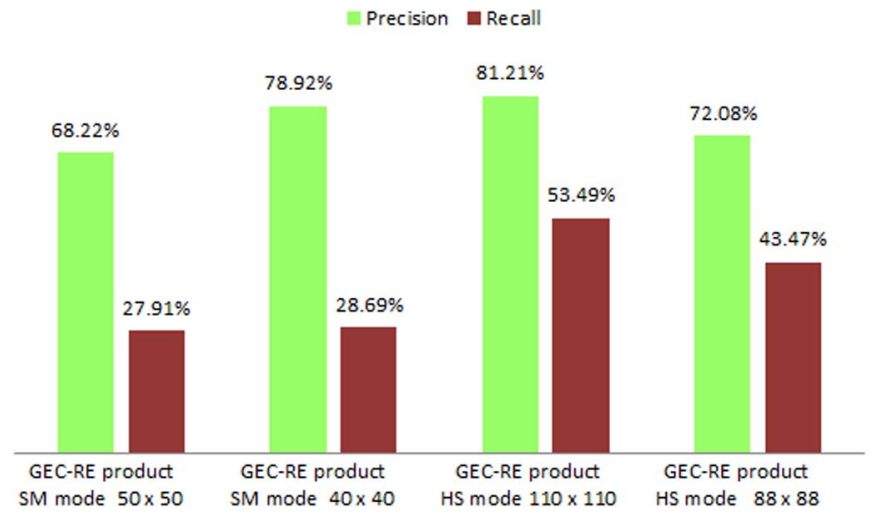

Fig. 21. Per-product classification accuracies as average over all the classes and algorithms which are available in test data set-2.
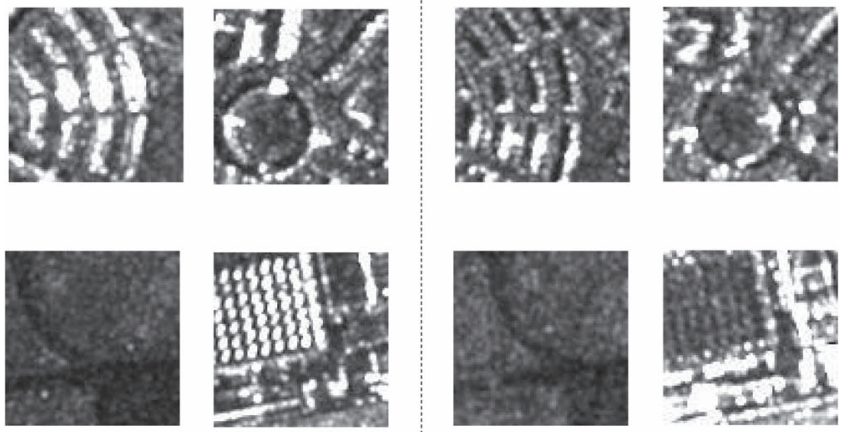

Fig. 22. Examples of different patches tiled from the TerraSAR-X GEC-RE product with SM mode, having (left side) descending looking and (right side) ascending looking.

$40 \times 40$ pixels, we computed the "per-class" classification accuracy. The results (see Fig. 23) are presented comparatively for each class, and two PFs are presented for both the ascending and the descending looking.

In Fig. 24, the "per-feature" classification accuracies of each algorithm for all classes in the case of the ascending and the descending looking are presented. The average of the precision/recall over all classes and features for the ascending looking is $85.71 \%$ and $61.74 \%$, respectively, and is higher than the descending looking (precision is $76.37 \%$, and recall is $51.43 \%$ ).

Comparing the ascending and the descending looking of TerraSAR-X GEC-RE product with SM mode, we can conclude the following.

1) For all the classes (water, vegetation, urban, etc.), the precision/recall per class and $\mathrm{PF}$ are better when the data have been acquired with the ascending looking. The number of retrieved and annotated classes for the Berlin area is equal to 17 (see Fig. 17).

2) For the ascending orbit direction, clear results are obtained for the classes containing structures/buildings and vegetation in the case of precision. Regarding the recall, aside from the previously listed classes, the water class can be added.

Note that, when the products with different orbit looking are compared, we should keep in mind that it is not possible to

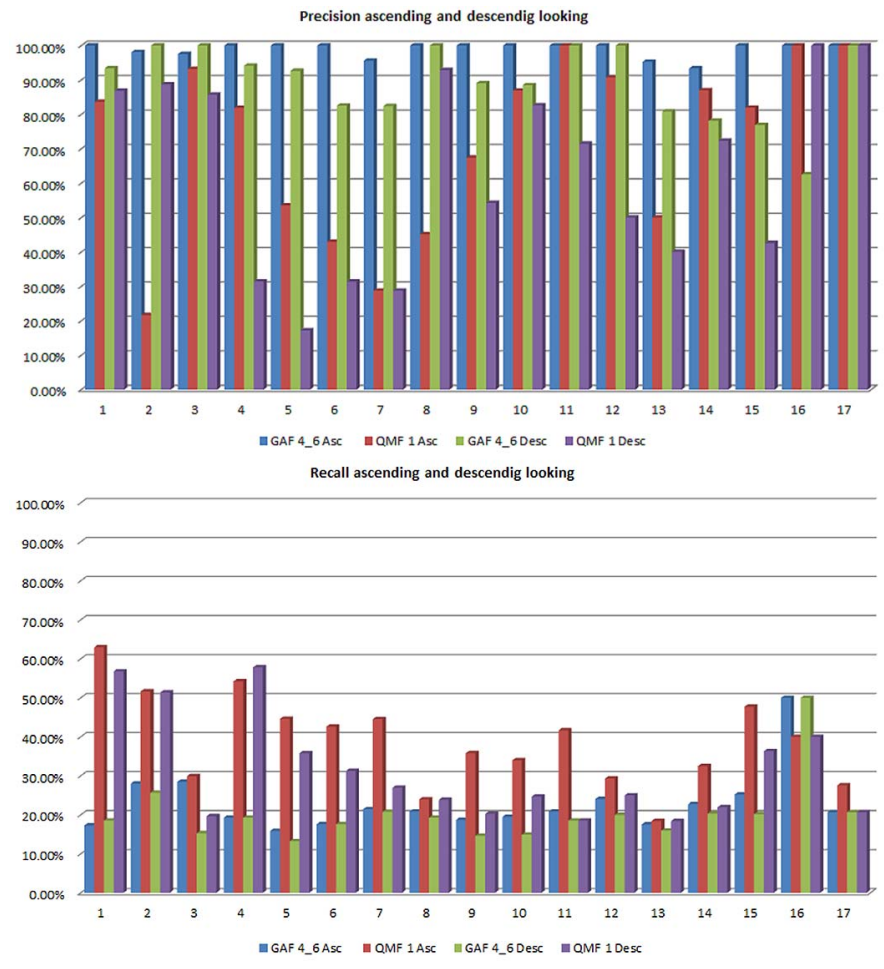

Fig. 23. Per-class classification accuracies of the GAFs (with 4 scales and 6 orientations) and QMFs (with number of levels equal to 1 ) for Berlin in the case of GEC-RE product with SM mode with a patch size of $40 \times 40$ pixels (ascending (Asc) looking and descending (Desc) looking).

$$
\text { Precision Recall }
$$

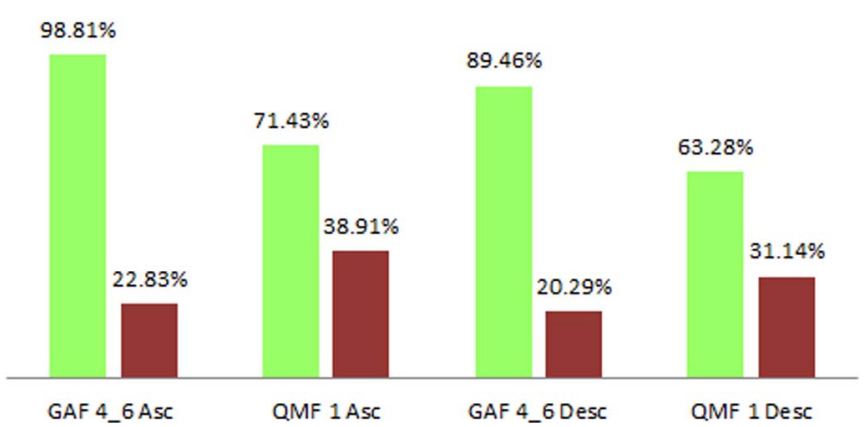

Fig. 24. Per-feature classification accuracies as average over all classes of the TerraSAR-X GEC-RE product with SM mode with ascending (Asc) and descending (Desc) available in test data set-2.

have always the same incidence angle or other parameters (e.g., resolution, pixel spacing, etc.) of these products that cover the same area.

One of the reason for which the product with the ascending looking is more accurate in classification than that with the descending looking can be the parameters (e.g., resolution, incidence angle, etc.) of the data that influence the accuracy of the classification. The influence of the incidence angle is studied further in the next section.

\section{Dependence of the SAR Imaging Incident Angle}

There are some applications that are sensitive to the incidence angle (e.g., urban cartography). Our objective in this 

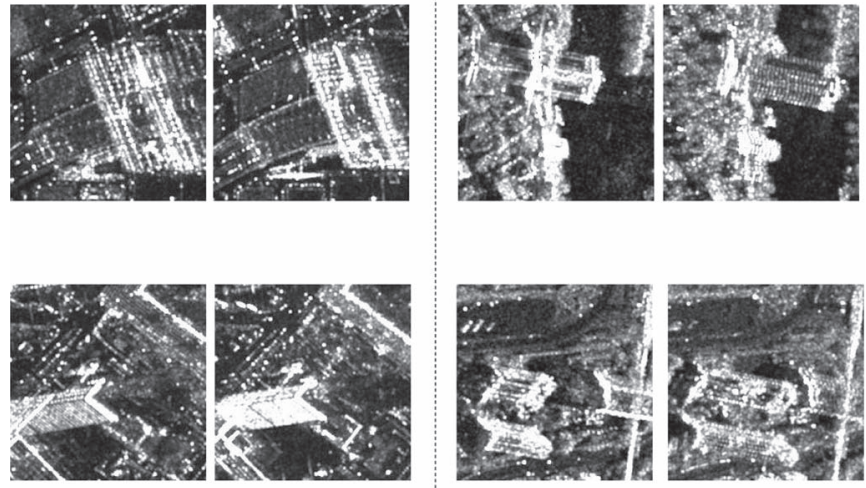

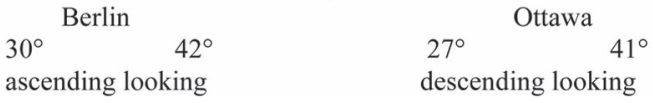

Fig. 25. Examples of different patches tiled from the TerraSAR-X GEC-RE product image with HS mode, having different incidence angles for Berlin and Ottawa.

section is to find the influence of the incidence angle of the TerraSAR-X product in classification accuracy.

The Berlin and Ottawa sites from test data set-2 were considered. The acquired products from the TerraSAR-X archive are the GEC-RE products with HS, having the incidence angles of $30^{\circ}$ and $42^{\circ}$, respectively, with ascending looking for Berlin and incidence angles of $27^{\circ}$ and $41^{\circ}$, respectively, with descending looking for Ottawa.

In order to find the difference between the incidence angles close to the lower bound and other close to the upper bound of the sensor range in Fig. 25, some examples for Berlin and Ottawa are presented.

The methodology of evaluation is similar with the one used in the previous section and detailed in Section III. The images are tiled into patches of $220 \times 220$ pixels (subsampled after that to $110 \times 110$ pixels), extracting the GAFs and QMFs for each patch and incidence angle.

The next step is to classify and to compute the classification accuracy for each site and incidence angle. The results of this evaluation are presented in Fig. 26 for the Berlin area (ascending looking) and Fig. 27 for the Ottawa area (descending looking).

In Fig. 26, the results of precision show a better distribution for the incidence angle equal to $30^{\circ}$ in the case of GAFs, contrary with the recall where the better distribution of the results is for $42^{\circ}$ in the case of QMFs.

For the second site, Ottawa, in Fig. 27, the precision/recall of each class is displayed for GAFs and QMFs when the image was acquired with an incidence angle equal to $27^{\circ}$ and $41^{\circ}$, having the descending looking. The results in precision are better for a lower value of the incidence angle (e.g., $27^{\circ}$ ), and those in recall are better for an upper value of the incidence angle (e.g., $41^{\circ}$ ). For both sites, the GAFs and QMFs were applied to the data.

In first case, we have the incidence angle closer to the lower bound of the satellite range (precision), and in the second case, we have the incidence angle closer to the upper bound of the satellite range (recall). In Fig. 28, the average of the classification accuracy is shown.
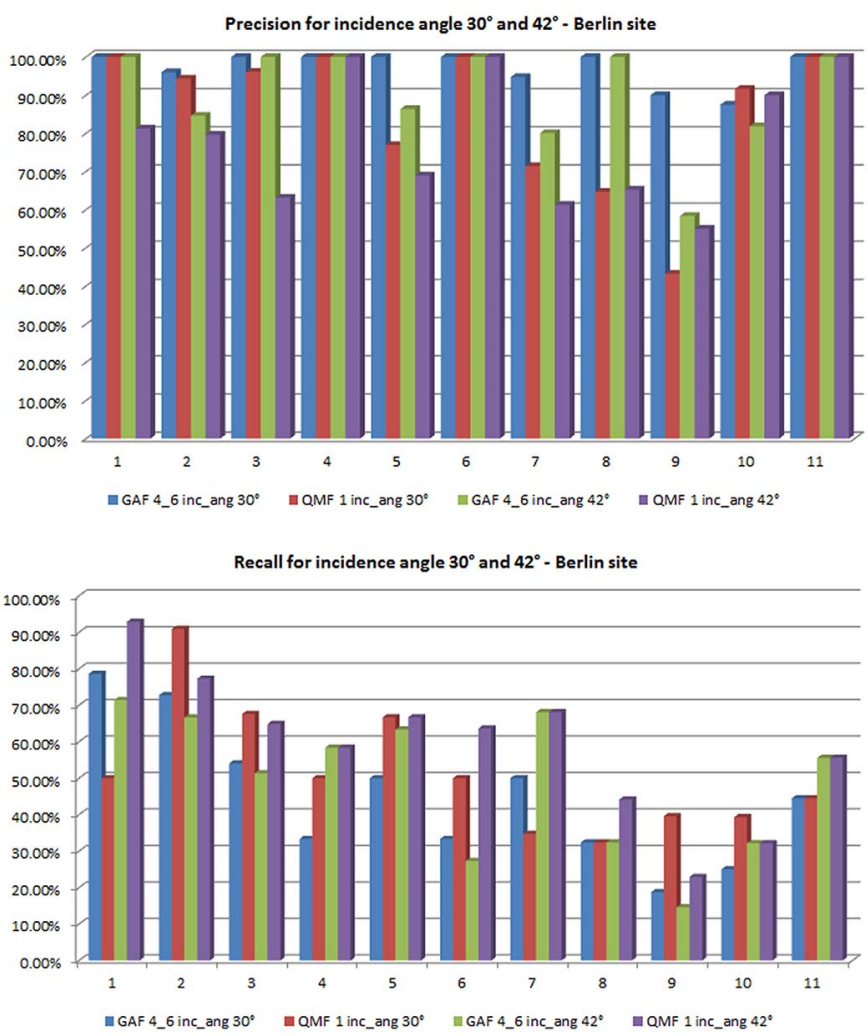

Fig. 26. Per-class classification accuracies (precision—top; recall—bottom) of GAFs (with 4 scales and 6 orientations) and QMFs (with number of levels equal to 1) for Berlin in the case of GEC-RE product with HS mode, ascending looking, and incidence angles of $30^{\circ}$ and $42^{\circ}$.
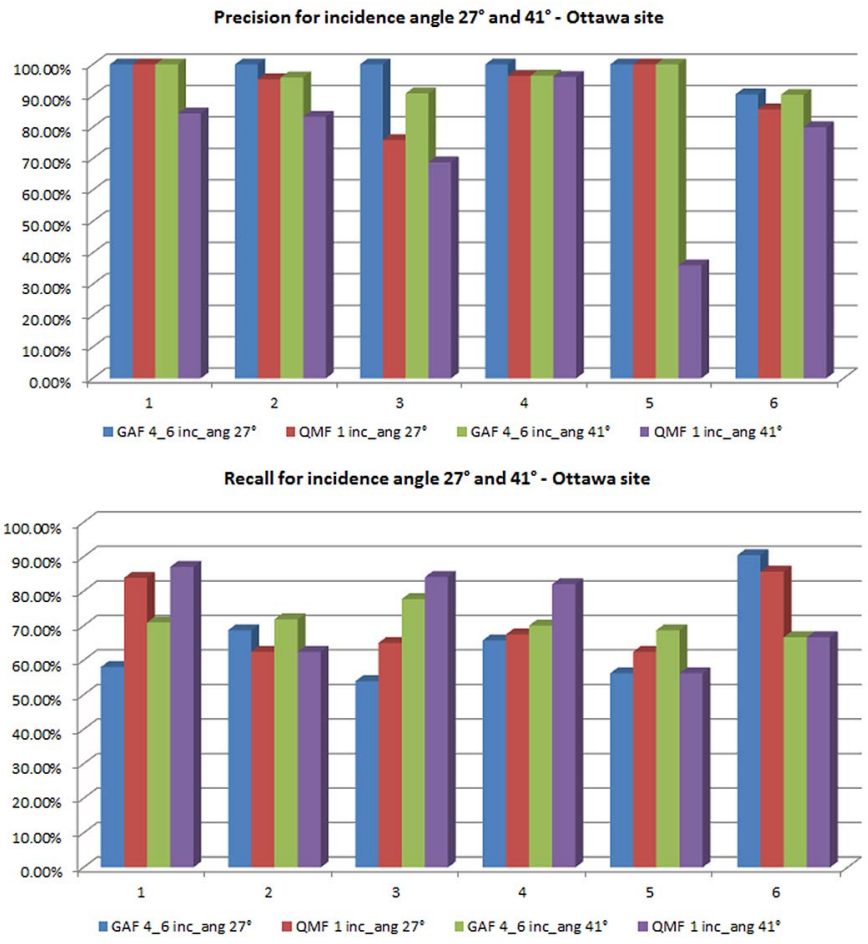

Fig. 27. Per-class classification accuracies (precision—top; recall—bottom) of GAFs (with 4 scales and 6 orientations) and QMFs (with number of levels equal to 1) for Ottawa in the case of GEC-RE product with HS mode, descending looking, and incidence angles of $27^{\circ}$ and $41^{\circ}$. 


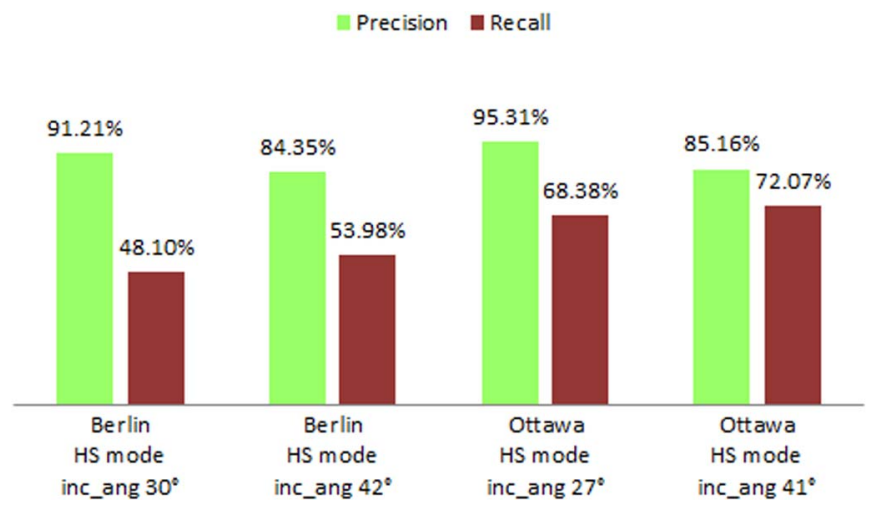

Fig. 28. Per-incidence angle classification accuracies as average over all retrieved classes of the TerraSAR-X GEC-RE product with HS mode and different incidence angle (inc_ang) available in test data set-2 for Berlin and Ottawa.

\begin{tabular}{|c|c|c|c|c|c|}
\hline Water & Chanel & $\begin{array}{l}\text { Building } \\
\text { Reflection }\end{array}$ & $\begin{array}{l}\text { Urban } \\
\text { type } 1\end{array}$ & $\begin{array}{l}\text { Urban } \\
\text { type } 2\end{array}$ & Field \\
\hline 31 & 32 & 37 & 111 & 16 & 42 \\
\hline
\end{tabular}

Fig. 29. Typical classes (patch size of $110 \times 110$ ) extracted from Ottawa (TerraSAR-X GEC-RE product with HS mode).

The discussion reached a point when a decision must be taken; we need precision that means the accuracy of the relevant patches from the total number of retrieved patches or recall that means more relevant patches to be retrieved from the data set. The objective of our system is to annotate large data sets (hundreds of sites) by grouping the similar patches in classes using the proposed methodology, and recall can be considered as a parameter for assessing the accuracy of classification.

Taking into account the recall, we have better classification accuracy for an incidence angle closer to the upper bound of the sensor range, $42^{\circ}$ (for the Berlin site) and $41^{\circ}$ (for the Ottawa site), respectively. This remark is valid for both the ascending and the descending looking: ascending looking in the case of Berlin and descending looking in the case of Ottawa.

Note that, for the TerraSAR-X product with the HS mode, the sensor range (incidence angle) is between $20^{\circ}$ and $55^{\circ}$.

Another remark that can be notice here is that the results of the incidence angle need to be associated with their specific orbit looking, and this means an ascending looking for the Berlin site and a descending looking for the Ottawa site.

The number of retrieved classes is equal to 11 for Berlin (see Fig. 17) and to 6 for Ottawa (see Fig. 29), and the total of the patches is 375 for each site.

The good classes in recall that we identified during the evaluation are as follows:

1) for Berlin: forest, forest plus other objects, building reflection, and urban;

2) for Ottawa: water, channel, building reflection, urban, and field.

\section{E. Conclusion}

After the investigation of the dependence on the patch size, orbit direction, and incident angle, we can conclude the following.

1) The optimal patch size is related to the resolution and product/mode type and is $40 \times 40$ pixels for the GEC$\mathrm{RE}$ product with the $\mathrm{SM}$ mode and $110 \times 110$ pixels for the GEC-RE product with the HS mode. The accuracy of classification is higher for products having the HS mode than the SM mode.

2) The ascending orbit performs better in the accuracy of classification than the descending looking with a remark that we must consider the influence of the resolution of the product and the incidence angle.

3) The upper value of the incidence angle (e.g., $41^{\circ}$ or $42^{\circ}$ ) has better classification accuracy than the lower value of the incidence angle (e.g., $27^{\circ}$ or $30^{\circ}$ ). This is valid for both orbit looking, ascending and descending.

\section{CONClusion And Future Work}

In this paper, we investigated the dependence of the products, PF extracted from the data, and parameters of the data: resolution, pixel spacing, patch size, orbit direction, and incidence angle. Each product was selected in order to answer the question "which is the dependence between the product/data and PFs or parameters."

As a general conclusion, we can say that the optimal product for signal processing and, therefore, for feature extraction is the MGD product. The results of the classification accuracy (the precision/recall) demonstrate that the GAFs and QMFs with different configurations give better results compared with the other investigated algorithms.

Regarding the parameters of the product (e.g., patch size, orbit direction, and incidence angle), the GEC product is more appropriate to be used for this evaluation because this product has a better accuracy of the geolocation of the image or can be used for image coregistration.

A direct comparison between MGD and GEC products is very difficult to be realized, and in terms of image contents, this is changing inside of the patch from one product to another.

The selected product modes are the SM mode because it is the most known mode for SAR and the HS mode because it is the most used mode for research.

As geometric resolution configuration, the RE seems to be the best based on the obtained results.

For all tiled patches, approximately 21000 patches, an annotation is done, and a semantic meaning is chosen based on the predominant content of the patch.

In terms of product parameters, the optimal patch size is $40 \times 40$ pixels for the SM mode and $110 \times 110$ pixels for the HS mode, where, for both cases, the patch is subsampled before being used. Regarding the resolution, an accurate classification is obtained for data having about a $6.5-\mathrm{m}$ resolution in the case of the SM mode and $2.9 \mathrm{~m}$ in the case of the HS mode. 


\section{No. of sites aquired with ascending and descending looking}

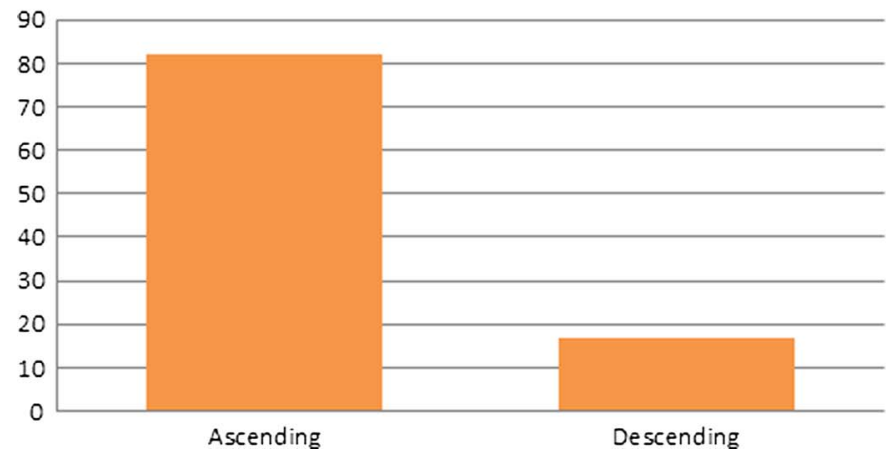

No. of sites aquired with different incidence angle

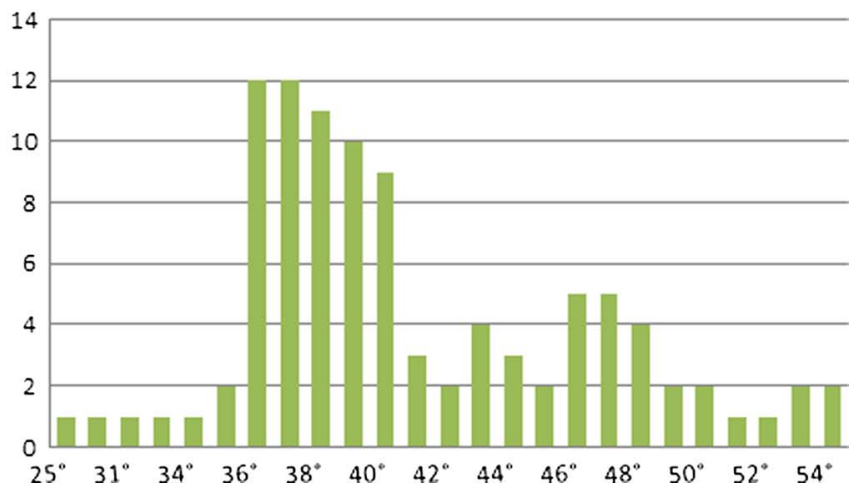

Fig. 30 Statistic over the parameters of the data (orbit direction and incidence angle) used for further evaluation. The sites are acquired over the world (Africa, Asia, Europe, Middle East, North America, and South America), and their characteristics are as follows: TerraSAR-X multilook ground detected products, RE geometric resolution configuration, and HS mode.

For the orbit direction, the ascending looking is more suitable for classification than the descending looking, but we need to consider also the resolution and incidence angle of the product.

Finally, taking into account the last parameter investigated, the incidence angle, the accuracy of the classification (the recall) shows that the value of the incidence angle closer to the upper bounds of the sensor range is better than the lower value of this.

The applicability of the experiments may result in a number of applications.

1) for urban cartography, the TerraSAR-X products with the HS mode, having the incidence angle close to the upper bound of the TerraSAR-X sensor range. This application is sensitive to the incidence angle.

2) for forest monitoring, the TerraSAR-X with the HS mode combining the lower and upper bounds of the TerraSAR-X sensor range.

3) for hydrology monitoring, the TerraSAR-X products with the SM mode, having the orbit with the ascending looking and invariability to the incidence angle.

4) for PGS archive, the developed algorithms and the results can be used as a "module" that can be integrated in the new project Earth Observation image Librarian (EOLib) [5] which is the next generation of IIM system. EOLib will be operated in the PGS of TerraSAR-X/TanDEM-X, Sentinel 1, etc.

The diversity of parameters involved (e.g., product/type and its parameters, $\mathrm{PF}$ algorithms, and its parameters) is rather high; however, the analysis and results reported in [2], [20], [21], [29], and [30] allow a good generalization.

The results obtained during this analysis are confirmed by the new evaluation which has the objective of the semantic annotation of a large TerraSAR-X data set [21] and not the accuracy of the classification (precision/recall) as in this paper. Hundreds of sites over the world were processed, and about 90000 patches were annotated. In the next figure (see Fig. 30), a statistic over the two parameters of the enhanced data set, namely, orbit looking and incidence angle, is presented. As can be seen here in this figure, more than $80 \%$ of the sites are with the ascending looking or with the value of the incidence angle closer to the upper bound of the sensor range. As a future work, we intend to use this annotated enhanced data set to evaluate other parameters of the data, PFs, or classification algorithms.

\section{REFERENCES}

[1] TX-GS-DD-3302 TerraSAR-X Basic Products Specification Document, no. 1.6, TX-GS-DD-3302.

[2] C. O. Dumitru, M. Datcu, M. Koubarakis, M. Sioutis, and B. Nikolaou, "Ontologies for the virtual observatory for TerraSAR-X data," TELEIOS, Athens, Greece, Rep. D6.2.1-TELEIOS, 2012.

[3] J. Anderson, E. Hardy, J. Roach, and R. Witmer, A land use and land cover classification system for use with remote sensor data, United States Government Printing Office, Washington, DC, USA. [Online]. Available: http://landcover.usgs.gov/pdf/anderson.pdf

[4] M. Wolfmueller, D. Dietrich, E. Sireteanu, S. Kiemle, E. Mikusch, and M. Boettcher, "Data flow and workflow organization-The data management for the TerraSAR-X payload ground segment," IEEE Trans. on Geoscience and Remote Sensing, vol. 47, no. 1-1, pp. 44-50, Jan. 2009.

[5] Earth Observation Image Librarian-EOLib Project. [Online]. Available: http://deepenandlearn.esa.int/tiki-index.php?page=EOLIB+Project

[6] GeoTIFF Format. [Online]. Available: http://www.remotesensing.org/ geotiff/geotiff.html

[7] H. Breit, M. Eineder, T. Fritz, B. Schattler, M. Huber, and J. Mittermayer, "TerraSAR-X products and product performance update," in Proc. IEEE IGARSS, Denver, CO, USA, 2006, pp. 1921-1921.

[8] TerraSAR-X Products Tree. [Online]. Available: http://bprc.osu.edu/ rsl/GIIPSY/documents/9_DLR-stg_ipy_SARworkshop_TSX systemcapabilities_DF.pdf

[9] C.-R. Shyu, M. Klaric, G. J. Scott, and W. K. Mahamaneerat, "Knowledge discovery by mining association rules and temporal-spatial information from large-scale geospatial image databases," in Proc. IEEE IGARSS, Denver, CO, USA, 2006, pp. 17-20.

[10] C.-R. Shyu, M. Klaric, G. Scott, A. Barb, C. Davis, and K. Palaniappan, "GeoIRIS: Geospatial information retrieval and indexing systemContent mining, semantics modeling, and complex queries," IEEE Trans. Geosci. Remote Sens., vol. 45, no. 4, pp. 839-852, Apr. 2007.

[11] A. Popescu, I. Gavat, and M. Datcu, "Contextual descriptors for scene classes in very high resolution SAR images," IEEE Geosci. Remote Sens. Lett., vol. 9, no. 1, pp. 80-84, Jan. 2012.

[12] P. Birjandi and M. Datcu, "Patch contextual descriptors for very high resolution satellite images: A topographic ICA approach," IEEE Geosci. Remote Sens. Lett., to be published.

[13] GLCM Tutorial. [Online]. Available: http://www.fp.ucalgary.ca/ mhallbey/orderliness_group.htm

[14] R. Haralick, K. Shanmugam, and I. Dinstein, "Textural features for image classification," IEEE Trans. Syst., Man, Cybern., vol. SMC-3, no. 6, pp. 610-621, Nov. 1973.

[15] F. Albregtsen, "Statistical texture measures computed from gray level coocurrence matrices," University of Oslo, Oslo, Norway, 2008, Image Processing Laboratory. 
[16] B. S. Manjunath and W. Y. Ma, "Texture features for browsing and retrieval of image data," IEEE Trans. Pattern Anal. Mach. Intell., vol. 18, no. 8, pp. 837-842, Aug. 1996.

[17] E. P. Simoncelli and E. H. Adelson, "Non-separable extensions of quadrature mirror filters to multiple dimensions," Proc. IEEE, vol. 78, no. 4, pp. 652-664, Apr. 1990.

[18] A. Popescu, I. Gavat, and M. Datcu, "Complex SAR image characterization using space variant spectral analysis," in Proc. IEEE Radar Conf., 2008, pp. 1-4.

[19] I. Dimitrovski, S. Loskovska, and I. Chorbev, "Efficient content-based image retrieval using support vector machines for feature aggregation," Proc. Innov. Comput. Sci. Softw. Eng., pp. 319-324, 2010.

[20] C. O. Dumitru, J. Singh, and M. Datcu, "Selection of relevant features and TerraSAR-X products for classification of high resolution SAR images," in Proc. EUSAR, 2012, pp. 243-246.

[21] Virtual Observatory Infrastructure for Earth Observation DataTELEIOS Project. [Online]. Available: http://www.earthobservatory.eu/ knowledge-discovery-from-EO-data

[22] B. Liu, C. Zhu, K. Wang, X. Liu, and W. Yu, "A one-class-extraction framework for high resolution SAR image classification," in Proc. IEEE CIE Int. Conf., 2011, pp. 732-735.

[23] C. Zhu, B. Liu, Q. Yu, X. Liu, and W. Yu, "A spy positive and unlabeled learning classifier and its application in HR SAR image scene interpretation," in Proc. IEEE Radar Conf., 2012, pp. 0516-0521.

[24] C.-C. Chang and C.-J. Lin, LIBSVM-A Library for Support Vector Machines. [Online]. Available: http://www.csie.ntu.edu.tw/ cjlin/libsvm/

[25] T. Fritz, H. Breit, and M. Eineder, "TerraSAR-X products-Tips and tricks," in Proc. TerraSAR-X Sci. Meeting, Oberpfaffenhofen, Germany, 2008. [Online]. Available: http://terrasar-x.dlr.de/papers_sci_meet_3/ tricks/TSX-products_tips_and_tricks-1.pdf

[26] M. Costache, H. Maitre, and M. Datcu, "Categorization based relevance feedback search engine for Earth observation images repositories," in Proc. IEEE IGARSS, Denver, CO, USA, 2006, pp. 13-16.

[27] MPEG 7. [Online]. Available: http://mpeg.chiariglione.org/standards/ mpeg-7/mpeg-7.htm

[28] P. Vaidyanathan, "Quadrature mirror filter banks, M-band extensions and perfect-reconstruction techniques," IEEE ASSP Mag., vol. 4, no. 3, pp. 420, Jul. 1987.

[29] C. O. Dumitru and M. Datcu, "Dependency of SAR image structure descriptors with incidence angle," in Proc. 4th Int. Conf. Adv. SPACOMM, Chamonix, France, 2012, pp. 92-97.

[30] C. O. Dumitru and M. Datcu, "Study and assessment of selected primitive features behavior for SAR image description," in Proc. IEEE IGARSS, Munich, Germany, 2012, pp. 3596-3599.

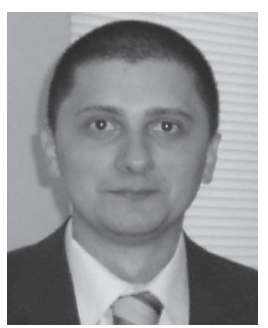

Corneliu Octavian Dumitru received the B.S. and M.S. degrees in applied electronics from the Faculty of Electronics, Telecommunications and Information Technology, Politehnica University Bucharest, Bucharest, Romania, in 2001 and 2002, respectively, the Ph.D. degree in engineering from Politehnica University Bucharest in 2006, and the Ph.D. degree in telecommunications from Pierre and Marie Curie University, Paris, France, in 2010.

At the Politehnica University, he had a teaching activity as a Lecturer, delivering lectures and seminaries and supervising laboratory works in the fields of information and estimation theory, communication theory, and signal processing. From 2005 to 2006 and in 2008, he was a coordinator for two national grants delivered by the Romanian Ministry of Education and Research. Since 2010, he has been a Scientist with the Remote Sensing Technology Institute (IMF), Earth Observation Center (EOC), German Aerospace Center (DLR), Oberpfaffenhofen, Germany. His research interest is in stochastic process information, model-based sequence recognition and understanding, basics of man-machine communication, information managing, and retrieval in extended databases. Recently, he is involved in several projects in the frame of the European Satellite Agency and FP7 Programs for information extraction, taxonomies, and data mining using remote sensing imagery.

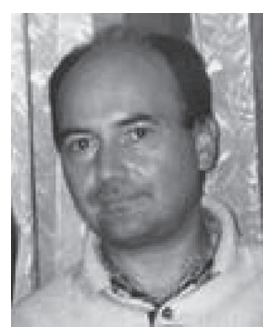

Mihai Datcu (SM'04-F'13) received the M.S. and $\mathrm{Ph} . \mathrm{D}$. degrees in electronics and telecommunications from the University Politehnica of Bucharest (UPB), Bucharest, Romania, in 1978 and 1986, respectively. In 1999, he received the title "Habilitation à diriger des recherches" in computer science from University Louis Pasteur, Strasbourg, France.

Since 1981, he has been a Professor with the Faculty of Electronics, Telecommunications and Information Technology, UPB, working in signal/image processing and electronic speckle interferometry. Since 1993, he has been a Scientist with the German Aerospace Center (DLR), Oberpfaffenhofen, Germany. He is developing algorithms for analyzing very high resolution synthetic aperture radar (SAR) and interferometric SAR data. He is engaged in research related to information theoretical aspects and semantic representations in advanced communication systems. Currently, he is a Senior Scientist and Image Analysis Research Group Leader with the Remote Sensing Technology Institute of DLR, Oberpfaffenhofen. Since 2011, he is also leading the Immersive Visual Information Mining research laboratory at the Munich Aerospace Faculty and is the Director of the Research Center for Spatial Information at UPB. He has held Visiting Professor appointments with the University of Oviedo, Oviedo, Spain, University Louis Pasteur and the International Space University in Strasbourg, France, the University of Siegen, Siegen, Germany, the University of Camerino, Camerino, Italy, and the Swiss Center for Scientific Computing, Manno, Switzerland. From 1992 to 2002, he had a longer Invited Professor assignment with the Swiss Federal Institute of Technology, ETH Zurich. Since 2001, he has initiated and leaded the Competence Centre on Information Extraction and Image Understanding for Earth Observation, at ParisTech, Telecom Paris, a collaboration of DLR with the French Space Agency (CNES). He has been a Professor holder of the DLR-CNES Chair at ParisTech, Telecom Paris. His research interests are in information and complexity theory, stochastic processes, Bayesian inference, and image information mining (IIM). He and his team have developed and are currently developing the operational IIM processor in the payload ground segment systems for the German missions TerraSAR-X, TanDEM-X, and the European Satellite Agency Sentinels 1 and 2. He is the author of more than 200 scientific publications, among them are about 50 journal papers and a book on number theory.

Prof. Datcu is a member of the European Image Information Mining Coordination Group and of the Data Archiving and Distribution Technical Committee of the IEEE Geoscience and Remote Sensing Society. 